\title{
THE STRUCTURE OF SOME EQUIVARIANT THOM SPECTRA
}

\author{
STEVEN R. COSTENOBLE
}

\begin{abstract}
We show that the equivariant Thom spectra $\mathrm{MO}_{\mathrm{Z}_{2}}$ and $\mathrm{mO}_{\mathrm{Z}_{2}}$ do not split as wedges of equivariant Eilenberg-Mac Lane spectra, as they do nonequivariantly. This is done by finding two-stage Postnikov towers giving these spectra, and determining the nontrivial $k$-invariants. We also consider the question: In what sense is the spectrum $\mathrm{mO}_{\mathbf{Z}_{2}}$ representing unoriented bordism unique?
\end{abstract}

\section{INTRODUCTION}

In [6] it was shown that, for groups of odd order, the unoriented equivariant Thom spectra decompose as wedges of Eilenberg-Mac Lane spectra, just as in the nonequivariant case. One of our goals in this paper is to show that this is not true when the group is the two-element group $\mathbf{Z}_{2}$. In fact, we show, in Theorems 3.8 and 4.5, that the two common versions of the Thom spectrum, $\mathrm{MO}_{\mathrm{z}_{2}}$ and $\mathrm{mO}_{\mathrm{z}_{2}}$ (defined in $\S \S 3$ and 4), have nontrivial two-stage Postnikov towers, and we determine the $k$-invariants.

Of course, in order to talk about equivariant $k$-invariants we must know something about equivariant Eilenberg-Mac Lane spectra and maps between them. $\S 2$ is devoted to studying the cases that we need, and in particular we compute there some of the simpler equivariant cohomology operation groups.

In $\S 4$ we raise the question of the uniqueness of the spectrum representing equivariant geometric bordism, and give several answers. In $\S 5$ we study certain equivariant transfers, in order to give a more satisfactory uniqueness result than we get in $\S 4$.

\section{Preliminaries}

We start with some general results on the structure of $\mathbf{Z}_{2}$-spectra. Throughout this paper we will use the categories of equivariant spectra developed in [10]; much of this section is a summary of results proved there, particularly in its §II.9.

The basic construction that we wish to examine is this: Recall that there is a $\mathrm{Z}_{2}$-space $E \mathrm{Z}_{2}$ which has a free $\mathbf{Z}_{2}$-action, and is contractible when we forget

1980 Mathematics Subject Classification (1985 Revision). Primary 55P91; Secondary 55P42, 55S45, 55S91, 57R85. 
the $\mathbf{Z}_{2}$-action. Let $E \mathbf{Z}_{2}^{+}$denote this space with a disjoint $\mathbf{Z}_{2}$-fixed basepoint attached. There is a unique nontrivial based map $E \mathbf{Z}_{2}^{+} \rightarrow S^{0}$, where $S^{0}$ is the two-point space with trivial $\mathbf{Z}_{2}$-action, and we let $\widetilde{E Z}_{2}$ be the cofiber of this map. If $Y$ is any $Z_{2}$-spectrum, we get the cofibration sequence

$$
E \mathbf{Z}_{2}^{+} \wedge Y \rightarrow Y \rightarrow \widetilde{E \mathbf{Z}}_{2} \wedge Y \text {. }
$$

In this section we will look at the two outside terms of this sequence, and show that they are determined by certain nonequivariant spectra depending on $Y$. This has the effect of reducing the study of $Y$ to nonequivariant questions. In particular, this will be the starting point of the study of the spectrum $M O_{\mathrm{z}_{2}}$. In $\S 3$ we will see that this cofibration sequence, when $Y$ is $M O_{\mathrm{z}_{2}}$, is almost the two-stage Postnikov tower that we are looking for.

Now for some details. Let $L$ denote the nontrivial one-dimensional real representation of $\mathbf{Z}_{2}$, and $\mathbf{R}$ the trivial one. Let $\mathscr{U}=\mathbf{R}^{\infty} \oplus L^{\infty}$ be a complete $\mathbf{Z}_{2}$-universe (in the language of [10]). Then we have the category $\mathbf{Z}_{2} \mathscr{S} \mathscr{U}$ of $\mathbf{Z}_{2}$-spectra indexed on $\mathscr{U}$. We also have the categories $\mathscr{S} \mathscr{U}$ and $\mathscr{S} \mathscr{U}^{\mathbf{Z}_{2}}$, of nonequivariant spectra indexed on $\mathscr{U}$ and $\mathscr{U}^{\mathbf{Z}_{2}}$ respectively, where $\mathscr{U}^{\mathbf{Z}_{2}}=\mathbf{R}^{\infty}$.

There are various functors relating these categories. Let e denote the trivial group, and let $p: \mathbf{Z}_{2} \rightarrow \mathbf{e}$ be the unique map. Then there is an induced functor

$$
p^{*}: \mathscr{S} \mathscr{U}^{\mathbf{Z}_{2}} \rightarrow \mathbf{Z}_{2} \mathscr{S} \mathscr{U}
$$

which gives a nonequivariant spectrum the trivial $\mathbf{Z}_{2}$-action, and extends its grading to all of $\mathscr{U}$. This functor has a right adjoint

$$
(-)^{\mathbf{Z}_{2}}: \mathbf{Z}_{2} \mathscr{S} \mathscr{U} \rightarrow \mathscr{S} \mathscr{U}^{\mathbf{Z}_{2}},
$$

which restricts the indexing to $\mathscr{U}^{\mathbf{Z}_{2}}$ and then takes spacewise fixed-points. On the other hand, there is the inclusion of the identity, $i: \mathbf{e} \rightarrow \mathbf{Z}_{2}$ which induces a functor $i^{*}: \mathbf{Z}_{2} \mathscr{S} \mathscr{U} \rightarrow \mathscr{S} \mathscr{U}$; this functor merely forgets the $\mathbf{Z}_{2}$-action.

We also need to relate the categories $\mathscr{S} \mathscr{U}$ and $\mathscr{S} \mathscr{U}^{\mathbf{Z}_{2}}$. Let $\alpha: \mathscr{U}^{\mathbf{Z}_{2}} \rightarrow \mathscr{U}$ be the inclusion. Then, as explained in [10, §II.1], we have a pair of adjoint equivalences $\alpha^{*}: \mathscr{S} \mathscr{U} \rightarrow \mathscr{S} \mathscr{U}^{\mathbf{Z}_{2}}$ and $\alpha_{*}: \mathscr{S} \mathscr{U}^{\mathbf{Z}_{2}} \rightarrow \mathscr{S} \mathscr{U}$. Therefore, we will adopt an abuse of language and consistently confuse $\mathscr{S} \mathscr{U}$ and $\mathscr{S} \mathscr{U}^{\mathrm{Z}_{2}}$. For example, from the definitions it is easy to see that $i^{*} p^{*} X=\alpha_{*} X$ for any nonequivariant spectrum $X$. Therefore, we will say that $i^{*} p^{*} X$ is naturally equivalent to $X$. Again, for a $Z_{2}$-spectrum $Y$, the adjunctions give us a natural map $\alpha_{*}\left(Y^{\mathbf{Z}_{2}}\right)=i^{*} p^{*}\left(Y^{\mathbf{Z}_{2}}\right) \rightarrow i^{*} Y$, so we will say that there is a natural transformation $Y^{\mathbf{Z}_{2}} \rightarrow i^{*} Y$, which we think of as inclusion of the fixed-points.

Let us begin studying the cofibration $(*)$ by studying $\widetilde{E \mathbf{Z}}_{2} \wedge Y$. Define a functor

$$
\varphi: \mathbf{Z}_{2} \mathscr{S} \mathscr{U} \rightarrow \mathscr{S} \mathscr{U}^{\mathbf{Z}_{2}}
$$

by $\varphi(Y)=\left(\widetilde{E \mathbf{Z}}_{2} \wedge Y\right)^{\mathbf{Z}_{2}}$. For many purposes this functor has better properties than the fixed-point functor (see [1, §7, and 10, §II.9]). 
Theorem 1.1. If $X$ is a nonequivariant $C W$-spectrum, there is a natural equivalence $X \simeq \varphi p^{*} X$, the map being the one adjoint to $p^{*} X \rightarrow \widetilde{E Z}_{2} \wedge p^{*} X$. If $Y$ is a $\mathbf{Z}_{2}-C W$-spectrum, there is a natural equivalence

$$
\widetilde{E \mathbf{Z}}_{2} \wedge p^{*} \varphi Y \simeq \widetilde{E \mathbf{Z}}_{2} \wedge Y
$$

the map being induced by the adjoint of the identity map $\varphi Y \rightarrow \varphi Y$.

These statements are special cases of II.9.10 and II.9.11 of [10].

Theorem 1.2. If $X$ and $Y$ are $\mathrm{Z}_{2}-C W$-spectra, then

$$
\left[X, \widetilde{E Z}_{2} \wedge Y\right]_{\mathbf{Z}_{2}} \cong\left[\widetilde{E Z}_{2} \wedge X, \widetilde{E Z}_{2} \wedge Y\right]_{\mathbf{Z}_{2}} \cong[\varphi X, \varphi Y]
$$

where $[-,-]_{\mathbf{Z}_{2}}$ denotes $\mathbf{Z}_{2}$-homotopy classes of $\mathbf{Z}_{2}$-maps, and $[-,-]$ denotes nonequivariant homotopy classes of nonequivariant maps.

The first isomorphism comes from II.9.2 of [10], and the second is immediate from Theorem 1.1.

These two results allow us to reduce questions about the spectrum $\widetilde{E Z_{2}} \wedge Y$ to nonequivariant ones. In fact, they say that the category of such spectra is equivalent to the nonequivariant category, via $\varphi$. (Again, this is a special case of II.9.6 of [10].)

Now we turn to spectra of the form $E Z_{2}^{+} \wedge Y$.

Theorem 1.3. Suppose that $f: X \rightarrow Y$ is a map of $\mathbf{Z}_{2}-C W$-spectra, such that $i^{*} f: i^{*} X \rightarrow i^{*} Y$ is an equivalence. Then $1 \wedge f: E \mathbf{Z}_{2}^{+} \wedge X \rightarrow E \mathbf{Z}_{2}^{+} \wedge Y$ is a $\mathbf{Z}_{2}$-equivalence.

This follows from II.2.2 of [10].

To further understand $E Z_{2}^{+} \wedge Y$, we introduce the notion of a split $\mathbf{Z}_{2}$ spectrum. Recall from [11] that a $Z_{2}$-spectrum $Y$ is said to be split if there is a nonequivariant map $\xi: i^{*} Y \rightarrow Y^{Z_{2}}$, such that the composite of $\xi$ with inclusion of the fixed points, $i^{*} Y \rightarrow Y^{Z_{2}} \rightarrow i^{*} Y$, is (homotopic to) the identity. By adjunction, a splitting gives us a map $\tilde{\xi}: p^{*} i^{*} Y \rightarrow Y$.

Lemma 1.4. If $Y$ is a split $\mathbf{Z}_{2}$-spectrum, then $i^{*} \tilde{\xi}: i^{*} p^{*} i^{*} Y \rightarrow i^{*} Y$ is an equivalence.

The proof just involves checking the definitions and adjunctions involved.

Theorem 1.5. If $Y$ is a split $\mathbf{Z}_{2}$-spectrum, then there is a natural equivalence

$$
E \mathbf{Z}_{2}^{+} \wedge Y \simeq E \mathbf{Z}_{2}^{+} \wedge p^{*} i^{*} Y \text {. }
$$

This follows immediately from Theorem 1.3 and Lemma 1.4.

Again, this says that $E \mathbf{Z}_{2}^{+} \wedge Y$ is determined by the nonequivariant spectrum $i^{*} Y$. Putting together Theorems 1.1 and 1.5, we get the following theorem. 
Theorem 1.6. If $Y$ is a split $\mathbf{Z}_{2}$-spectrum, then there is a (co)fibration sequence

$$
E \mathbf{Z}_{2}^{+} \wedge p^{*} i^{*} Y \rightarrow Y \rightarrow \widetilde{E Z}_{2} \wedge p^{*} \varphi Y
$$

Thus, to understand the structure of $Y$, it suffices to understand the nonequivariant spectra $i^{*} Y$ and $\varphi Y$, and the connecting map

$$
\widetilde{E \mathbf{Z}_{2}} \wedge p^{*} \varphi Y \rightarrow \Sigma E \mathbf{Z}_{2}^{+} \wedge p^{*} i^{*} Y \text {. }
$$

Finally, let us record some more facts about split spectra. First we introduce a notation we will use throughout the rest of the paper. If $X$ is a $\mathbf{Z}_{2}$-spectrum or a nonequivariant spectrum, we write $\widetilde{X}_{*}(-)$ for the reduced homology theory on based $Z_{2}$-spaces or spaces that the spectrum represents, and $\widetilde{X}^{*}(-)$ for the cohomology theory. (We will always index on the integers.) Similarly, write $X_{*}(-)$ and $X^{*}(-)$ for the theories on spectra. Write $X_{*}=\tilde{X}_{*}\left(S^{0}\right)$ and $X^{*}=\tilde{X}^{*}\left(S^{0}\right)$, so $X^{n}=X_{-n}$.

Recall the following facts about nonequivariant spectra.

Theorem 1.7. If $X$ and $Y$ are nonequivariant $C W$-spectra, and $f: \tilde{X}_{*}(A) \rightarrow$ $\tilde{Y}_{*}(A)$ is a natural transformation of homology theories on finite $C W$-complexes $A$, then $f$ is represented by a map of spectra $X \rightarrow Y$.

Corollary 1.8. If $X$ and $Y$ are nonequivariant $C W$-spectra such that the homology theories they represent are naturally equivalent on finite $C W$-complexes, then $X$ and $Y$ are equivalent.

The proofs of these results are standard applications of Spanier-Whitehead duality, Milnor's $\lim ^{1}$ exact sequence, and the Whitehead Theorem. We have stated these results both because we will use them, and because we want to point out that they are not true in the equivariant context, at least if we insist on using integer grading. Examples of this are easy to find among the EilenbergMac Lane spectra that we will look at in the next section; we will give another example in bordism later. We can fix this by using $R O(G)$-grading, but this is not always a natural thing to do. For example, bordism is most naturally graded only on the integers.

One observation we make from Theorem 1.7 is that we can tell that a $\mathbf{Z}_{2}$ spectrum $Y$ is split by noticing the algebraic fact that $\widetilde{Y}_{*}(A) \rightarrow \widetilde{i}^{*} Y_{*}(A)$ is a naturally split epimorphism for all $\mathrm{Z}_{2}$-trivial finite $\mathrm{CW}$-complexes $A$. For example, this is easy to see for bordism theories.

If $X$ is a $Z_{2}$-ring spectrum, we require that any splitting be a map of ring spectra. With this we can show

Theorem 1.9. Suppose that $X$ is a split $\mathbf{Z}_{2}$-ring spectrum, that $Y$ is a $\mathbf{Z}_{2}$ spectrum, and that $(Y \wedge X)^{*}$ is a flat $\left(i^{*} X\right)^{*}$-module. Then for finite nonequivariant $C W$-spectra $A$ we have a natural isomorphism

$$
\left(i^{*} X\right)^{*}(A) \otimes_{\left(i^{*} X\right)^{*}}(Y \wedge X)^{*} \cong(Y \wedge X)^{*}\left(p^{*} A\right) .
$$

Moreover, this isomorphism is also natural in $Y$. 
Proof. The transformation is defined in the obvious way, as the composite

$$
\begin{aligned}
{\left[A, i^{*} X\right]^{*} \otimes[S, Y \wedge X]_{\mathbf{Z}_{2}}^{*} } & \rightarrow\left[p^{*} A, p^{*} i^{*} X\right]_{\mathbf{Z}_{2}}^{*} \otimes[S, Y \wedge X]_{\mathbf{Z}_{2}}^{*} \\
& \stackrel{\tilde{\xi}}{\rightarrow}\left[p^{*} A, X\right]_{\mathbf{Z}_{2}}^{*} \otimes[S, Y \wedge X]_{\mathbf{Z}_{2}}^{*} \\
& \rightarrow\left[p^{*} A, Y \wedge X \wedge X\right]_{\mathbf{Z}_{2}}^{*} \rightarrow\left[p^{*} A, Y \wedge X\right]_{\mathbf{Z}_{2}}^{*}
\end{aligned}
$$

This is clearly natural in $A$ and $Y$. Moreover, if we let $A=S$, we see that it is an isomorphism. The flatness assumption assures us that we are comparing cohomology theories, so we conclude that the map is an isomorphism for any finite $A$.

Corollary 1.10. Suppose that $X$ is a split $\mathbf{Z}_{2}$-ring spectrum, that $Y$ is a $\mathbf{Z}_{2}$ spectrum, and that $(Y \wedge X)^{*}$ is a free $\left(i^{*} X\right)^{*}$-module. Then $(Y \wedge X)^{\mathbf{Z}_{2}}$ is a wedge of suspensions of $i^{*} X$, and the conclusion of Theorem 1.9 holds for any nonequivariant $C W$-spectrum $A$.

Proof. Combine Theorem 1.9 and the easier cohomological version of Corollary 1.8 .

\section{EILENBERG-MAC LANE SPECTRA}

In order to describe an equivariant Postnikov tower, we need to know something about the equivariant analogues of Eilenberg-Mac Lane spectra, and something about maps between them. In this section we look at the cases that we need for our study of Thom spectra.

Let us write $\mathrm{HZ}_{2}$ for the nonequivariant Eilenberg-Mac Lane spectrum with $\pi_{0} H \mathrm{Z}_{2}=\mathrm{Z}_{2}$ and $\pi_{n} H \mathrm{Z}_{2}=0$ for $n \neq 0$. This spectrum represents ordinary homology and cohomology with $Z_{2}$ coefficients. If we omit the coefficients below, this is the theory we mean.

To specify a nonequivariant Eilenberg-Mac Lane spectrum we need to specify only a single group, but for the equivariant analogue we need more: we need to specify a Mackey functor (see, for example, [9]). Recall that if $X$ is a $\mathbf{Z}_{2}$ spectrum, there is a Mackey functor $\underline{\pi}_{n}(X)$ which has

$$
\underline{\pi}_{n}(X)\left(\mathbf{Z}_{2} / \mathbf{Z}_{2}\right)=\pi_{n}\left(X^{\mathbf{Z}_{2}}\right) \cong\left[S^{n}, X\right]_{\mathbf{Z}_{2}}
$$

and

$$
\underline{\pi}_{n}(X)\left(\mathbf{Z}_{2} / \mathbf{e}\right)=\pi_{n}\left(i^{*} X\right) \cong\left[S^{n}, \mathbf{Z}_{2}^{+} \wedge X\right]_{\mathbf{Z}_{2}}
$$

The structure maps are the "restriction," induced by the inclusion of the fixedpoints, $X^{\mathbf{Z}_{2}} \rightarrow i^{*} X$, and the "transfer," induced by the equivariant projection $\mathbf{Z}_{2}^{+} \wedge X \rightarrow X$. Given a Mackey functor $M$, the corresponding $\mathbf{Z}_{2}$-EilenbergMac Lane spectrum, which we will call $H M$, is the $\mathrm{Z}_{2}-\mathrm{CW}$-spectrum (unique up to equivalence) with

$$
\underline{\pi}_{n}(H M) \cong \begin{cases}M, & n=0 \\ 0, & n \neq 0\end{cases}
$$


as Mackey functors. The equivariant homology and cohomology theories that these spectra represent are Bredon's ordinary theories, as extended to the stable context in [9]. We denote these theories by $H_{*}^{\mathrm{Z}_{2}}(-; M)$ and $H_{\mathbf{Z}_{2}}^{*}(-; M)$.

There are several Mackey functors that we will be interested in. Let $\underline{\mathbf{Z}}_{2}$ be the functor with $\underline{\mathbf{Z}}_{2}\left(\mathbf{Z}_{2} / \mathbf{Z}_{2}\right)=\mathbf{Z}_{2}=\underline{\mathbf{Z}}_{2}\left(\mathbf{Z}_{2} / \mathbf{e}\right)$, with the restriction map $\underline{\mathbf{Z}}_{2}\left(\mathbf{Z}_{2} / \mathbf{Z}_{2}\right) \rightarrow \underline{\mathbf{Z}}_{2}\left(\mathbf{Z}_{2} / \mathbf{e}\right)$ being the identity, and the transfer $\underline{\mathbf{Z}}_{2}\left(\mathbf{Z}_{2} / \mathbf{e}\right) \rightarrow$ $\underline{\mathbf{Z}}_{2}\left(\mathbf{Z}_{2} / \mathbf{Z}_{2}\right)$ being the zero map. Write $\overline{\mathbf{Z}}_{2}$ for the "dual" functor; again it has the value $Z_{2}$ at both orbits, but now the restriction is zero while the transfer is the identity. Let $\Phi$ be the functor with $\Phi\left(Z_{2} / Z_{2}\right)=Z_{2}$ but $\Phi\left(Z_{2} / \mathbf{e}\right)=0$, and let $\Omega$ be defined by $\Omega\left(Z_{2} / Z_{2}\right)=0$ while $\Omega\left(Z_{2} / \mathbf{e}\right)=Z_{2}$. The corresponding Eilenberg-Mac Lane spectra are then $H \underline{\mathbf{Z}}_{2}, H \overline{\mathbf{Z}}_{2}, H \Phi$, and $H \Omega$. It is useful to know what the corresponding homology theories look like for $\Phi$ and $\Omega$, and they are

$$
\widetilde{H}_{*}^{\mathbf{Z}_{2}}(A ; \Phi) \cong \widetilde{H}_{*}\left(A^{\mathbf{Z}_{2}}\right) \quad \text { and } \quad \widetilde{H}_{*}^{\mathbf{Z}_{2}}(A ; \Omega) \cong \widetilde{H}_{*}\left(\left(A / \mathbf{Z}_{2}\right) / A^{\mathbf{Z}_{2}}\right)
$$

for any $\mathrm{Z}_{2}-\mathrm{CW}$-complex $A$.

We start by examining the fibration sequence $E \mathrm{Z}_{2}^{+} \wedge H M \rightarrow H M \rightarrow \widetilde{E \mathrm{Z}_{2}} \wedge$ $H M$ when $M$ is one of these Mackey functors.

Theorem 2.1. $H \Phi \simeq \widetilde{E Z_{2}} \wedge p^{*} H Z_{2}$.

Proof. Check the homotopy groups of $\widetilde{E Z}{ }_{2} \wedge p^{*} H Z_{2}$. By Theorem 1.1 and the fact that $\widetilde{E Z_{2}}$ is nonequivariantly contractible, they are the same as $H \Phi$.

This result completely determines the fibration sequence for $H \Phi$; in particular, it shows that $E \mathbf{Z}_{2}^{+} \wedge H \Phi$ is trivial, since $E \mathbf{Z}_{2}^{+} \wedge \widetilde{E Z_{2}}$ is $\mathrm{Z}_{2}$-contractible.

The following result shows that the Mackey functors $\Omega, \underline{\mathbf{Z}}_{2}$, and $\overline{\mathbf{Z}}_{2}$ are closely related. Because of this result, it often suffices to study just one of them. We will concentrate on $\Omega$ for the most part.

Lemma 2.2. $\Sigma^{L-1} H \overline{\mathbf{Z}}_{2} \simeq H \Omega \simeq \Sigma^{1-L} H \underline{\mathbf{Z}}_{2}$, and the equivalences are unique up to homotopy.

Proof. Again, check the homotopy groups. In fact, using an explicit cellular structure for $S^{b L}$ and the cellular definitions of Bredon homology and cohomology, we can compute

$$
\underline{\pi}_{a}\left(\Sigma^{-b L} H \Omega\right)= \begin{cases}\overline{\mathbf{Z}}_{2}, & a+b=0 \text { and } a<0, \\ \Omega, & a=b=0, \\ \underline{\mathbf{Z}}_{2}, & a+b=0 \text { and } a>0, \\ \Phi, & a+b>0 \text { and } a<0 ; \text { or } a+b<0 \text { and } a>0, \\ 0, & \text { otherwise. }\end{cases}
$$

Since there is only one nontrivial homomorphism $\Omega \rightarrow \Omega$, it follows (from the Universal Coefficient spectral sequence, for example) that there is (up to homotopy) only one nontrivial self-map $H \Omega \rightarrow H \Omega$. From this follows the stated uniqueness of the equivalences. 
Corollary 2.3. There is a unique nontrivial $\mathbf{Z}_{2}$-map $\theta: H \Omega \rightarrow \Sigma^{L-1} H \Omega$, and it is a nonequivariant equivalence.

Proof. There is a unique nontrivial map of Mackey functors $\Omega \rightarrow \underline{\mathbf{Z}}_{2}$, inducing $H \Omega \rightarrow H \underline{\mathrm{Z}}_{2} \cong \Sigma^{L-1} H \Omega . H \Omega \rightarrow H \underline{\mathrm{Z}}_{2}$ is clearly a nonequivariant equivalence.

We also note that suspension of $\theta$ gives a unique nontrivial map $H \underline{\mathbf{Z}}_{2} \rightarrow$ $\Sigma^{L-1} H \underline{\mathrm{Z}}_{2}$, which we will also call $\theta$.

Corollary 2.4. $\theta$ induces $\mathbf{Z}_{2}$-homotopy equivalences

$$
E \mathrm{Z}_{2}^{+} \wedge H \Omega \simeq \Sigma^{L-1} E \mathrm{Z}_{2}^{+} \wedge H \Omega
$$

and

$$
E \mathbf{Z}_{2}^{+} \wedge H \underline{\mathbf{Z}}_{2} \simeq \Sigma^{L-1} E \mathbf{Z}_{2}^{+} \wedge H \underline{\mathbf{Z}}_{2}
$$

Proof. Combine Corollary 2.3 and Theorem 1.3 for the first statement, and then use Lemma 2.2 to get the second by suspending.

Theorem 2.5. $E \mathrm{Z}_{2}^{+} \wedge H \Omega \simeq E \mathrm{Z}_{2}^{+} \wedge p^{*} H \mathrm{Z}_{2} \simeq E \mathrm{Z}_{2}^{+} \wedge H \underline{\mathbf{Z}}_{2}$.

Proof. Apply Theorem 1.5 to the spectrum $H \underline{Z}_{2}$, which is clearly split. Since $H \Omega \rightarrow H \underline{\mathrm{Z}}_{2}$ is a nonequivariant equivalence, we can use Theorem 1.3 to replace $\underline{\mathbf{Z}}_{2}$ with $\Omega$.

Theorem 2.6.

$$
\widetilde{E \mathbf{Z}_{2}} \wedge H \Omega \simeq \bigvee_{k=1}^{\infty} \Sigma^{k} H \Phi
$$

and

$$
\widetilde{E \mathbf{Z}}_{2} \wedge H \underline{\mathbf{Z}}_{2} \simeq \bigvee_{k=0}^{\infty} \Sigma^{k} H \Phi
$$

Moreover, these decompositions can be chosen so that the map $\widetilde{\mathrm{EZ}_{2}} \wedge H \Omega \rightarrow$ $\widetilde{E \mathbf{Z}}_{2} \wedge H \underline{\mathbf{Z}}_{2}$ is the obvious inclusion, and the maps

$$
\widetilde{E \mathbf{Z}}_{2} \wedge H \Omega \rightarrow \Sigma^{L-1} \widetilde{E \mathbf{Z}_{2}} \wedge H \Omega \simeq \Sigma^{-1} \widetilde{E \mathbf{Z}}_{2} \wedge H \Omega
$$

and

$$
\widetilde{E \mathbf{Z}}_{2} \wedge H \underline{\mathbf{Z}}_{2} \rightarrow \Sigma^{L-1} \widetilde{E \mathbf{Z}_{2}} \wedge H \underline{\mathbf{Z}}_{2} \simeq \Sigma^{-1} \widetilde{E \mathbf{Z}}_{2} \wedge H \underline{\mathbf{Z}}_{2}
$$

induced by $\theta$ are given by the obvious "shift of summands" maps.

Proof. Consider the map $p^{*} H \mathbf{Z}_{2} \rightarrow H \underline{\mathbf{Z}}_{2}$ adjoint to the equivalence $H \mathrm{Z}_{2} \simeq$ $\left(H \underline{\mathbf{Z}}_{2}\right)^{\mathbf{Z}_{2}}$. Using Theorem 1.1, it is not difficult to show that the composite $\varphi p^{*} H \mathbf{Z}_{2} \rightarrow \varphi H \underline{\mathbf{Z}}_{2} \rightarrow \varphi H \Phi$ is an equivalence, and so the composite $\widetilde{E \mathbf{Z}}_{2} \wedge$ $p^{*} H \mathrm{Z}_{2} \rightarrow{\widetilde{E Z_{2}}} \wedge H \underline{\mathbf{Z}}_{2} \rightarrow H \Phi$ is a $\mathrm{Z}_{2}$-equivalence. This splits off the lowest $H \Phi$, and in particular gives us a map $H \Phi \rightarrow \widetilde{E \mathbf{Z}}_{2} \wedge H \underline{\mathbf{Z}}_{2}$ which is an isomorphism on the lowest homotopy group.

Now consider the equivalence $H \underline{\mathbf{Z}}_{2} \simeq \Sigma^{L-1} H \Omega$ and the map $\theta: \widetilde{E Z}_{2} \wedge H \Omega \rightarrow$ $\widetilde{E \mathbf{Z}}{ }_{2} \wedge H \underline{\mathbf{Z}}_{2} \simeq \Sigma^{-1} \widetilde{E \mathbf{Z}}_{2} \wedge H \Omega$. Composing the map $H \Phi \rightarrow \widetilde{E \mathbf{Z}_{2}} \wedge H \underline{\mathbf{Z}}_{2} \simeq$ 
$\Sigma^{-1} \widetilde{E \mathbf{Z}_{2}} \wedge H \Omega$ with iterates of $\theta$ gives us a sequence of maps $\Sigma^{k} H \Phi \rightarrow \widetilde{E \mathbf{Z}_{2}} \wedge$ $H \underline{\mathbf{Z}}_{2}$, for $k \geq 0$, and $\Sigma^{k} H \Phi \rightarrow \widetilde{E \mathbf{Z}}_{2} \wedge H \Omega$, for $k \geq 1$. Together, these give us maps $\bigvee_{k=0}^{\infty} \Sigma^{k} H \Phi \rightarrow \widetilde{E \mathbf{Z}_{2}} \wedge H \underline{\mathbf{Z}}_{2}$ and $\bigvee_{k=1}^{\infty} \Sigma^{k} H \Phi \rightarrow \widetilde{E \mathbf{Z}}{ }_{2} \wedge H \Omega$, which are homotopy isomorphisms, hence homotopy equivalences. By construction, the several maps of the statement of this result respect these decompositions as claimed.

There is a characterization of the connecting map $\widetilde{E \mathbf{Z}_{2}} \wedge H \Omega \rightarrow \Sigma E \mathbf{Z}_{2}^{+} \wedge H \Omega$ which will be useful. Notice that this map is an equivalence on fixed-points, because $H \Omega^{\mathbf{Z}_{2}}$ is trivial.

Theorem 2.7. If $f: \bigvee_{k=1}^{\infty} \Sigma^{k} H \Phi \rightarrow \Sigma E \mathbf{Z}_{2}^{+} \wedge H \Omega$ is a $\mathbf{Z}_{2}$-map which is an isomorphism on fixed-point homotopy, then $f$ factors through the map $\widetilde{E \mathbf{Z}}{ }_{2} \wedge H \Omega \rightarrow$ $\Sigma E \mathbf{Z}_{2}^{+} \wedge H \Omega$, and the resulting map $\bigvee_{k=1}^{\infty} \Sigma^{k} H \Phi \rightarrow \widetilde{E Z}_{2} \wedge H \Omega$ is an equivalence. Proof. To get that $f$ factors as claimed, note that the composite of $f$ with the map $\Sigma E \mathbf{Z}_{2}^{+} \wedge H \Omega \rightarrow H \Omega$ must be trivial. This is true for dimensional reasons: there are no nontrivial maps $\Sigma^{k} H \Phi \rightarrow H \Omega$ for $k \geq 0$ (consider the Universal-Coefficients spectral sequence, for example). Once we know that $f$ factors as desired, the map into $\widetilde{E Z}_{2} \wedge H \Omega$ will be a homotopy isomorphism by our assumptions, so will be an equivalence.

Now we turn to the study of equivariant cohomology operations. Let $\mathscr{A}_{2}^{*}$ denote the nonequivariant mod 2 Steenrod algebra. We will grade maps between spectra cohomologically, so that a map $X \rightarrow Y$ of degree $n$ is the same as a map $X \rightarrow \Sigma^{n} Y$ of degree 0 . We will confine ourselves to integer grading (but see the comment following the next result).

Theorem 2.8. $[H \Phi, H \Phi]_{\mathrm{Z}_{2}}^{*} \cong \mathscr{A}_{2}^{*}$.

Proof.

$$
\begin{aligned}
{[H \Phi, H \Phi]_{\mathbf{Z}_{2}}^{*} } & \cong\left[\widetilde{E \mathbf{Z}_{2}} \wedge p^{*} H \mathbf{Z}_{2}, \widetilde{E \mathbf{Z}_{2}} \wedge p^{*} H \mathbf{Z}_{2}\right]_{\mathbf{Z}_{2}}^{*} \\
& \cong\left[H \mathbf{Z}_{2}, H \mathbf{Z}_{2}\right]^{*} \cong \mathscr{A}_{2}^{*}
\end{aligned}
$$

by Theorems $2.1,1.2$, and 1.1 .

Notice that the restriction to integer grading is not really important here, since $H \Phi \simeq \Sigma^{L} H \Phi$. For the same reason, all of our integer-graded results involving $H \Phi$ gives us $R O\left(\mathbf{Z}_{2}\right)$-graded results, if we want them.

Let $X$ be any $\mathbf{Z}_{2}$-space or spectrum. $\theta$ induces functions

$$
\begin{aligned}
& \theta:[H \Phi, X \wedge H \Omega]_{\mathbf{Z}_{2}}^{n} \rightarrow[H \Phi, X \wedge H \Omega]_{\mathbf{Z}_{2}}^{n-1} \\
& \theta:\left[H \Phi, X \wedge H \underline{\mathbf{Z}}_{2}\right]_{\mathbf{Z}_{2}}^{n} \rightarrow\left[H \Phi, X \wedge H \underline{\mathbf{Z}}_{2}\right]_{\mathbf{Z}_{2}}^{n-1},
\end{aligned}
$$

given by $[H \Phi, X \wedge H \Omega]_{\mathbf{Z}_{2}}^{n} \rightarrow\left[H \Phi, \Sigma^{L-1} X \wedge H \Omega\right]_{\mathbf{Z}_{2}}^{n} \cong\left[H \Phi, \Sigma^{-1} X \wedge H \Omega\right]_{\mathrm{Z}_{2}}^{n}$ using the fact that $H \Phi \simeq \Sigma^{L} H \Phi$ (the second version of $\theta$ is defined in the same way). 
Lemma 2.9. $\left[H \Phi, \widetilde{E \mathbf{Z}_{2}} \wedge H \underline{\mathrm{Z}}_{2}\right]_{\mathrm{Z}_{2}}^{*} \cong \mathscr{A}_{2}^{*}[[\theta]]$.

Note. $\mathscr{A}_{2}^{*}$ acts on $\left[H \Phi, \widetilde{E Z}_{2} \wedge H \underline{Z}_{2}\right]_{\mathrm{Z}_{2}}^{*}$ via maps $H \Phi \rightarrow H \Phi$, and $\theta$ acts as defined above. Therefore, this isomorphism is to be understood as an isomorphism of $\mathscr{A}_{2}^{*}[[\theta]]$-modules, where $\mathscr{A}_{2}^{*}[[\theta]]$ denotes the ring of homogeneous formal power series in $\theta$ with coefficients in $\mathscr{A}_{2}^{*}$. This makes sense because the degree of $\theta$ is -1 .

Proof. . This follows from Theorems 2.8 and 2.6. Notice that Theorem 2.6 says that $\theta$ is given by the evident shift operation when we use that decomposition of $\widetilde{E \mathbf{Z}_{2}} \wedge H \underline{\mathbf{Z}}_{2}$.

Theorem 2.10.

$$
\left[H \Phi, E \mathbf{Z}_{2}^{+} \wedge H \Omega\right]_{\mathbf{Z}_{2}}^{*} \cong \mathscr{A}_{2}^{*}\left[\left[\theta, \theta^{-1}\right]\right]
$$

and

$$
[H \Phi, H \Omega]_{\mathbf{Z}_{2}}^{*} \cong \mathscr{A}_{2}^{*}\left\{\sigma_{1}, \sigma_{2}, \ldots\right\}
$$

where $\sigma_{i}$ is the image of $\theta^{-i}$.

Again, these are to be understood as isomorphisms of modules. The free module over $\mathscr{A}_{2}^{*}$ with basis consisting of elements called $\sigma_{i}$ for $i \geq 1$ is denoted by $\mathscr{A}_{2}^{*}\left\{\sigma_{1}, \sigma_{2}, \ldots\right\}$.

Proof. Consider the fibration sequence $E \mathbf{Z}_{2}^{+} \wedge H \Omega \rightarrow H \Omega \rightarrow \widetilde{E Z}_{2} \wedge H \Omega$, and the resulting long exact sequence involving maps from $H \Phi$ into these spectra.

$[H \Phi, H \Omega]_{\mathrm{Z}_{2}}^{n}=0$ for $n<0$ by connectedness. The long exact sequence says then that

$$
\begin{aligned}
{\left[H \Phi, E \mathbf{Z}_{2}^{+} \wedge H \Omega\right]_{\mathbf{Z}_{2}}^{n} } & \cong\left[H \Phi, \Sigma^{-1} \widetilde{E Z}_{2} \wedge H \Omega\right]_{\mathbf{Z}_{2}}^{n}, \quad n<0 \\
& \cong\left[H \Phi, \widetilde{E \mathbf{Z}}{ }_{2} \wedge H \underline{\mathbf{Z}}_{2}\right]_{\mathbf{Z}_{2}}^{n}
\end{aligned}
$$

On the other hand, $\theta$ is an isomorphism

$$
\left[H \Phi, E \mathbf{Z}_{2}^{+} \wedge H \Omega\right]_{\mathbf{Z}_{2}}^{n} \cong\left[H \Phi, E \mathbf{Z}_{2}^{+} \wedge H \Omega\right]_{\mathrm{Z}_{2}}^{n-1},
$$

as we see by Corollary 2.4. Combining this with the calculation of Lemma 2.9, we must have that $\left[H \Phi, E \mathbf{Z}_{2}^{+} \wedge H \Omega\right]_{\mathrm{Z}_{2}}^{*} \cong \mathscr{A}_{2}^{*}\left[\left[\theta, \theta^{-1}\right]\right]$ as stated. Here $\theta^{-1}$ is just the inverse of the isomorphism $\theta$.

This implies that the long exact sequence is actually a short exact sequence

$$
0 \rightarrow \mathscr{A}_{2}^{*}[[\theta]] \rightarrow \mathscr{A}_{2}^{*}\left[\left[\theta, \theta^{-1}\right]\right] \rightarrow[H \Phi, H \Omega]_{\mathrm{Z}_{2}}^{*} \rightarrow 0 .
$$

Therefore, as $\mathscr{A}_{2}^{*}$-modules, $[H \Phi, H \Omega]_{\mathrm{Z}_{2}}^{*} \cong \mathscr{A}_{2}^{*}\left\{\sigma_{1}, \sigma_{2}, \ldots\right\}$, where $\sigma_{i}$ is the image of $\theta^{-i}$.

We will use the last calculation of this theorem in the form

$$
[H \Phi, \Sigma H \Omega]_{\mathrm{Z}_{2}}^{*} \cong \mathscr{A}_{2}^{*}\left[\theta^{-1}\right]
$$

with a slight abuse of notation. 
We need a slightly more general form of this calculation. If $V$ is a $\mathbf{Z}_{2}$-vector space and $M$ is a Mackey functor, write $M \otimes V$ for the Mackey functor with $(M \otimes V)(G / H)=M(G / H) \otimes V$. If $V^{*}$ is a graded $\mathrm{Z}_{2}$-vector space, write $H\left(M \otimes V^{*}\right)$ for the spectrum $\bigvee_{k=-\infty}^{\infty} \Sigma^{k} H\left(M \otimes V^{-k}\right)$.

Corollary 2.11. If $V^{*}$ and $W^{*}$ are graded $\mathbf{Z}_{2}$-vector spaces, then

$$
\left[H\left(\boldsymbol{\Phi} \otimes V^{*}\right), E \mathbf{Z}_{2}^{+} \wedge H\left(\Omega \otimes W^{*}\right)\right]_{\mathbf{Z}_{2}}^{*} \cong \operatorname{Hom}_{\mathbf{Z}_{2}}^{*}\left(V^{*}, \mathscr{A}_{2}^{*} \widehat{\otimes} W^{*}\left[\left[\theta, \theta^{-1}\right]\right]\right)
$$

and

$$
\left[H\left(\Phi \otimes V^{*}\right), \Sigma H\left(\Omega \otimes W^{*}\right)\right]_{\mathrm{Z}_{2}}^{*} \cong \operatorname{Hom}_{\mathrm{Z}_{2}}^{*}\left(V^{*}, \mathscr{A}_{2}^{*} \widehat{\otimes} W^{*}\left[\left[\theta^{-1}\right]\right]\right)
$$

$(\widehat{\otimes}$ indicates the completed tensor product).

Proof. Write $H\left(\Phi \otimes V^{*}\right)$ as a wedge of suspensions of $H \Phi$, using a basis for $V^{*}$. Then a map out of $H\left(\Phi \otimes V^{*}\right)$ is completely determined by its restriction to each summand of $H \Phi$. The calculations follow easily from the previous calculation.

Another useful way of looking at the same argument is this. Any particular map $f: H\left(\Phi \otimes V^{*}\right) \rightarrow E Z_{2}^{+} \wedge H\left(\Omega \otimes W^{*}\right)$, say, is completely determined by the induced homomorphism $f_{*}$ as in

$$
\begin{array}{ccc}
{\left[H \Phi, H\left(\Phi \otimes V^{*}\right)\right]_{\mathbf{Z}_{2}}^{*}} & \cong & \mathscr{A}_{2}^{*} \widehat{\otimes} V^{*} \\
\downarrow f_{*} & & \downarrow f_{*} \\
{\left[H \Phi, E \mathbf{Z}_{2}^{+} \wedge H\left(\Omega \otimes W^{*}\right)\right]_{\mathbf{Z}_{2}}^{*}} & \cong & \mathscr{A}_{2}^{*} \widehat{\otimes} W^{*}\left[\left[\theta, \theta^{-1}\right]\right] .
\end{array}
$$

Since $f_{*}$ preserves the action of $\mathscr{A}_{2}^{*}$, it is completely determined by the adjoint vector-space homomorphism $V^{*} \rightarrow \mathscr{A}_{2}^{*} \widehat{\otimes} W^{*}\left[\left[\theta, \theta^{-1}\right]\right]$.

This result will be useful because the two-stage Postnikov towers we will be considering will have $k$-invariants of precisely this form: maps from $H\left(\Phi \otimes V^{*}\right)$ to $\Sigma H\left(\Omega \otimes W^{*}\right)$. Thus this calculation tells us what all the possible $k$-invariants are, and gives us a way of specifying a particular invariant.

Let us complete this preliminary look at these Postnikov towers with some comments on how the $k$-invariant can be determined computationally. The proof of Corollary 2.11 gives us one good way: Calculate the homomorphism induced by the $k$-invariant in the cohomology of the spectrum $H \Phi$. This will be our approach in studying $\mathrm{MO}_{\mathrm{z}_{2}}$ in the next section.

However, it is curious that there is a place where we will never be able to detect the $k$-invariant. That place is the homology of spaces. In this we part company rather radically with the nonequivariant world. Here is the precise result. We switch to homological grading (so $H\left(\Phi \otimes V_{*}\right)=\bigvee_{k=-\infty}^{\infty} \Sigma^{k} H\left(\Phi \otimes V_{k}\right.$ ), and so on).

Theorem 2.12. Suppose that $k: H\left(\Phi \otimes V_{*}\right) \rightarrow \Sigma H\left(\Omega \otimes W_{*}\right)$ is a $\mathbf{Z}_{2}$-map, and $X$ is its fiber. Then for any $\mathbf{Z}_{2}-C W$-complex, there is a naturally split short exact sequence

$$
0 \rightarrow \widetilde{H}_{*}^{\mathbf{Z}_{2}}(A ; \Omega) \otimes W_{*} \rightarrow \widetilde{X}_{*}(A) \rightarrow \widetilde{H}_{*}^{\mathbf{Z}_{2}}(A ; \Phi) \otimes V_{*} \rightarrow 0
$$


In other words, $\tilde{X}_{*}(A)$ is naturally isomorphic to the direct sum of the two outside terms. Thus neither the value $\tilde{X}_{*}(A)$, nor any map $\tilde{X}_{*}(A) \rightarrow \tilde{X}_{*}(B)$ induced by a space-level map $A \rightarrow B$, depends on $k$.

Proof. The short exact sequence above comes from the long exact sequence induced by the fibration sequence defining $X$. We show that we can define a natural splitting, which shows that it is short exact. Since $\widetilde{H}_{*}^{\mathrm{Z}_{2}}\left(A^{\mathrm{Z}_{2}} ; \Omega\right)=0$ and $\widetilde{H}_{*}^{\mathrm{Z}_{2}}(A ; \Phi) \cong \widetilde{H}_{*}^{\mathrm{Z}_{2}}\left(A^{\mathrm{Z}_{2}} ; \Phi\right)$, we have a natural isomorphism

$$
\widetilde{H}_{*}^{\mathbf{Z}_{2}}(A ; \Phi) \otimes V_{*} \cong \widetilde{X}_{*}\left(A^{\mathbf{Z}_{2}}\right) \text {. }
$$

Combining this with the natural map $\tilde{X}_{*}\left(A^{\mathbf{Z}_{2}}\right) \rightarrow \tilde{X}_{*}(A)$ gives the splitting.

This simple observation has some interesting consequences for equivariant bordism, which we will return to in $\S 4$.

To end this section, let us look at one more group of maps between spectra which we will need in the next section.

Theorem 2.13.

$$
\left[E \mathbf{Z}_{2}^{+} \wedge H \Omega, H \Omega\right]_{\mathbf{Z}_{2}}^{*} \cong\left[E \mathbf{Z}_{2}^{+} \wedge H \Omega, E \mathbf{Z}_{2}^{+} \wedge H \Omega\right]_{\mathbf{Z}_{2}}^{*} \cong \mathscr{A}_{2}^{*}\left[\theta^{-1}\right]
$$

The action of $\mathscr{A}_{2}^{*}$ is given via the equivalence $E \mathrm{Z}_{2}^{+} \wedge H \Omega \simeq E \mathrm{Z}_{2}^{+} \wedge p^{*} H \mathrm{Z}_{2}$.

Proof. The first isomorphism follows from Theorem 1.2, because the third term in the long exact sequence would be

$$
\left[E \mathbf{Z}_{2}^{+} \wedge H \Omega, \widetilde{E \mathbf{Z}}_{2} \wedge H \Omega\right]_{\mathbf{Z}_{2}}^{*} \cong\left[\widetilde{E Z}_{2} \wedge E \mathbf{Z}_{2}^{+} \wedge H \Omega, \widetilde{E Z}_{2} \wedge H \Omega\right]_{\mathbf{Z}_{2}}^{*}
$$

which is 0 because $\widetilde{E \mathbf{Z}_{2}} \wedge E \mathbf{Z}_{2}^{+}$is $\mathbf{Z}_{2}$-contractible.

Since $\left[p^{*} H \mathbf{Z}_{2}, H \Omega\right]_{\mathbf{Z}_{2}}^{*} \cong\left[H \mathbf{Z}_{2},(H \Omega)^{\mathbf{Z}_{2}}\right]^{*}=0$, we have

$$
\left[E \mathbf{Z}_{2}^{+} \wedge p^{*} H \mathbf{Z}_{2}, H \Omega\right]_{\mathbf{Z}_{2}}^{*} \cong\left[\Sigma^{-1} \widetilde{E \mathbf{Z}}{ }_{2} \wedge p^{*} H \mathbf{Z}_{2}, H \Omega\right]_{\mathbf{Z}_{2}}^{*}
$$

However, $\Sigma^{-1} \widetilde{E Z}_{2} \wedge p^{*} H Z_{2} \simeq \Sigma^{-1} H \Phi$, so the calculation follows from Theorem 2.10 .

Warning: the ring structure given by composition in

$$
\left[E \mathbf{Z}_{2}^{+} \wedge H \Omega, E \mathbf{Z}_{2}^{+} \wedge H \Omega\right]_{\mathbf{Z}_{2}}^{*}
$$

is not the polynomial structure suggested by this isomorphism of $\mathscr{A}_{2}^{*}$-modules. The actual multiplication is not important here, and will be dealt with in a future paper.

\section{THE STRUCTURE OF $M \mathrm{O}_{\mathrm{Z}_{2}}$}

Let $M O_{\mathrm{Z}_{2}}$ be the $\mathrm{Z}_{2}$-spectrum defined by letting $M O_{\mathrm{z}_{2}}(V)$ be the Thom space of the universal $Z_{2}$-vector bundle of dimension $|V|$, the dimension of $V$ (in the language of [10], this defines a prespectrum, and we really want $\mathrm{MO}_{\mathrm{z}_{2}}$ to be the spectrum it generates). In this section we show that, unlike the 
nonequivariant case, this spectrum is not the product of Eilenberg-Mac Lane spectra. We will, in fact, find a two-stage Postnikov tower for this spectrum, and determine the $k$-invariant, by studying and modifying the fibration sequence

$$
E \mathbf{Z}_{2}^{+} \wedge M O_{\mathbf{Z}_{2}} \rightarrow M O_{\mathbf{Z}_{2}} \rightarrow \widetilde{E \mathbf{Z}}_{2} \wedge M O_{\mathbf{Z}_{2}}
$$

We start by recalling some known results. First of all, we should comment that, while $M \mathrm{O}_{\mathrm{Z}_{2}}$ does not represent the usual bordism of $\mathrm{Z}_{2}$-manifolds, it does represent the stable bordism groups. These are the bordism groups of stable $\mathbf{Z}_{2}-$ manifolds, where a stable manifold is the equivalence class of a $Z_{2}$-map

$$
f:(M, \partial M) \rightarrow\left(D\left(L^{n}\right), S\left(L^{n}\right)\right),
$$

$M$ a $\mathbf{Z}_{2}$-manifold, where $f$ is considered to be equivalent to

$$
f \times 1:\left(M \times D\left(L^{m}\right), \partial\left(M \times D\left(L^{m}\right)\right)\right) \rightarrow\left(D\left(L^{n+m}\right), S\left(L^{n+m}\right)\right)
$$

for any $m$ (rounding corners as necessary). See [4 or 5] for a fuller account. The useful consequence of this is that geometric arguments and constructions originally given for the usual bordism groups can often be carried over with only small modifications to the stable bordism groups, and hence give results about $M O_{\mathrm{z}_{2}}$.

Write $M O$ for the nonequivariant Thom spectrum. We first see that $i^{*} M O_{\mathrm{z}_{2}}$ $=M O$, as is obvious from the definition. Next, notice that there is an easily

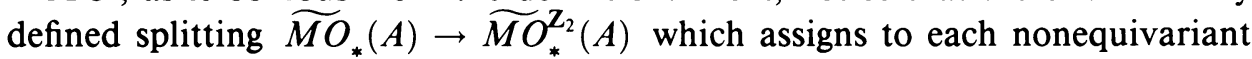
manifold over the $\mathrm{Z}_{2}$-trivial space $A$ the same manifold with trivial $\mathbf{Z}_{2}$-action. By Theorem 1.7, we conclude that $M O_{\mathrm{Z}_{2}}$ is a split ring spectrum.

Theorem 3.1. $\left(\widetilde{E Z}_{2} \wedge M O_{\mathbf{Z}_{2}}\right)^{*} \cong \mathscr{M}^{*}=M O^{*}\left[\beta_{1}^{-1}, \beta_{1}, \beta_{2}, \ldots\right]$ where $\left|\beta_{i}\right|=-i$ and the brackets denote the polynomial ring (with $\beta_{1}$ inverted). Also, $\left(E \mathbf{Z}_{2}^{+} \wedge\right.$ $\left.M O_{\mathrm{z}_{2}}\right)^{*} \cong M O^{*}\left\{r_{0}, r_{1}, \ldots\right\}$ where $\left|r_{i}\right|=-i$ and the braces indicate the free module with the indicated basis. The fibration sequence induces a short exact sequence

$$
0 \rightarrow M O_{\mathrm{Z}_{2}}^{*} \rightarrow \underline{\mathscr{M}}^{*} \stackrel{\partial}{\rightarrow} \Sigma^{-1} M O^{*}\left\{r_{0}, r_{1}, \ldots\right\} \rightarrow 0
$$

with $\partial\left(\beta_{1}^{n}\right)=\Sigma^{-1} r_{n-1}$ for $n \geq 1$. In particular, this shows that $M O_{\mathbf{Z}_{2}}^{*}$ is a free $M O^{*}$-module.

Proof. This is well known; see for example [2, 3, and 7]. We can apply the methods of the first two papers by noticing that their geometric arguments work perfectly well with stable bordism. In particular we can interpret $\left(E \mathbf{Z}_{2}^{+} \wedge M O_{\mathbf{Z}_{2}}\right)^{*}$ as bordism of free manifolds, and $\left(\widetilde{E Z}_{2} \wedge M O_{\mathrm{Z}_{2}}\right)^{*}$ as bordism of manifolds with free boundaries.

Corollary 3.2. $\widetilde{E Z}_{2} \wedge M O_{\mathrm{Z}_{2}} \simeq H\left(\Phi \otimes \underline{\mathscr{M}}^{*}\right)$.

Here we use the notation introduced before Corollary 2.11 . 
Proof. We use Corollary 1.10 (with $Y=\widetilde{E Z}_{2}$ and $X=M O_{\mathrm{z}_{2}}$ ) and Theorem 3.1 to show that $\varphi M O_{\mathrm{z}_{2}} \simeq H\left(\underline{\mathscr{M}}^{*}\right)$. Then apply Theorems 1.1 and 2.1 to get the result.

Recall that there is an equivalence $M O_{\mathrm{z}_{2}} \simeq \Sigma^{L-1} M O_{\mathrm{Z}_{2}}[7,5]$.

Corollary 3.3. $E \mathrm{Z}_{2}^{+} \wedge M O_{\mathrm{Z}_{2}} \simeq E \mathrm{Z}_{2}^{+} \wedge H\left(\Omega \otimes M O^{*}\right)$. Moreover, this equivalence can be chosen so that the equivalence

$$
\theta: E \mathbf{Z}_{2}^{+} \wedge H\left(\Omega \otimes M O^{*}\right) \simeq \Sigma^{L-1} E \mathbf{Z}_{2}^{+} \wedge H\left(\Omega \otimes M O^{*}\right)
$$

introduced in the last section agrees (up to homotopy) with the equivalence $\mathrm{EZ}_{2}^{+} \wedge$ $M \mathrm{O}_{\mathrm{Z}_{2}} \simeq \Sigma^{L-1} \mathrm{EZ}_{2}^{+} \wedge M \mathrm{O}_{\mathrm{Z}_{2}}$ mentioned above.

Proof. Using Theorem 1.5, we have that $E Z_{2}^{+} \wedge M O_{\mathrm{Z}_{2}} \simeq E Z_{2}^{+} \wedge p^{*} M O$. But $M O \simeq H\left(M O^{*}\right)$ [12], and $E \mathbf{Z}_{2}^{+} \wedge p^{*} H Z_{2} \simeq E \mathbf{Z}_{2}^{+} \wedge H \Omega$, hence the equivalance.

To see that the last part of the statement of this result is true for this decomposition, we first show that each summand of $E \mathbf{Z}_{2}^{+} \wedge H \Omega$ is carried to itself by the map $E \mathbf{Z}_{2}^{+} \wedge M O_{\mathbf{Z}_{2}} \rightarrow \Sigma^{L-1} E \mathbf{Z}_{2}^{+} \wedge M O_{\mathbf{Z}_{2}}$. Now, we can detect self-maps of $E \mathrm{Z}_{2}^{+} \wedge H \Omega$ by "probing" with $H \Phi$, by virtue of the equivalence $E \mathbf{Z}_{2}^{+} \wedge H \Omega \simeq E \mathbf{Z}_{2}^{+} \wedge p^{*} H \mathbf{Z}_{2}$, and the following commutative diagram:

$$
\begin{aligned}
& {\left[E \mathbf{Z}_{2}^{+} \wedge p^{*} H \mathbf{Z}_{2}, E \mathbf{Z}_{2}^{+} \wedge H \Omega\right]_{\mathbf{Z}_{2}}^{*} \rightarrow\left[\Sigma^{-1} \widetilde{E \mathbf{Z}}{ }_{2} \wedge p^{*} H \mathbf{Z}_{2}, E \mathbf{Z}_{2}^{+} \wedge H \Omega\right]_{\mathbf{Z}_{2}}^{*}} \\
& \downarrow \cong \downarrow \\
& {\left[E \mathbf{Z}_{2}^{+} \wedge p^{*} H_{\mathbf{Z}_{2}}, H \Omega\right]_{\mathbf{Z}_{2}}^{*} \quad \cong \quad\left[\Sigma^{-1} \widetilde{E \mathbf{Z}_{2}} \wedge p^{*} H \mathbf{Z}_{2}, H \Omega\right]_{\mathbf{Z}_{2}}^{*} \text {. }}
\end{aligned}
$$

The isomorphisms in the diagram were noted in Theorem 2.13. This diagram shows that the map across the top is injective, and in this sense we can detect any self-map of $E \mathbf{Z}_{2}^{+} \wedge H \Omega$ by probing with the map $\Sigma^{-1} H \Phi \simeq \Sigma^{-1} \widetilde{E Z}_{2} \wedge p^{*} H Z_{2} \rightarrow$ $E \mathbf{Z}_{2}^{+} \wedge p^{*} H \mathbf{Z}_{2} \simeq E \mathbf{Z}_{2}^{+} \wedge H \Omega$. Since $E \mathbf{Z}_{2}^{+} \wedge H \Omega \simeq \Sigma^{L-1} E \mathbf{Z}_{2}^{+} \wedge H \Omega$, the same is true for (integer graded) maps from $E \mathbf{Z}_{2}^{+} \wedge H \Omega$ to $\Sigma^{L-1} E \mathbf{Z}_{2}^{+} \wedge H \Omega$.

Now it happens that there is such a probe available in a natural way. Take a map $p^{*} M O \rightarrow M O_{\mathrm{z}_{2}}$ adjoint to a splitting. This gives us a commutative diagram

$$
\begin{array}{ccc}
\widetilde{E Z}_{2} \wedge p^{*} M O & \rightarrow \Sigma E \mathbf{Z}_{2}^{+} \wedge p^{*} M O \\
\downarrow & \downarrow \simeq \\
\widetilde{E Z}_{2} \wedge M O_{\mathbf{Z}_{2}} \rightarrow & \Sigma E \mathbf{Z}_{2}^{+} \wedge M O_{\mathbf{Z}_{2}} .
\end{array}
$$

In the decomposition $\widetilde{E \mathbf{Z}}_{2} \wedge M O_{\mathrm{Z}_{2}} \simeq H\left(\Phi \otimes \mathscr{M}^{*}\right), \widetilde{E \mathbf{Z}}_{2} \wedge p^{*} M O$ corresponds to the $M O^{*}$-multiples of the identity in $\mathscr{M}^{*}$. Following them in the diagram

$$
\begin{array}{ccc}
\widetilde{E Z}_{2} \wedge M O_{\mathbf{Z}_{2}} & \rightarrow \quad \Sigma E \mathbf{Z}_{2}^{+} \wedge M O_{\mathbf{Z}_{2}} \\
\Sigma^{L-1} \widetilde{E \mathbf{Z}}_{2} \wedge M O_{\mathbf{Z}_{2}} & \rightarrow \Sigma^{L} E \mathbf{Z}_{2}^{+} \wedge M O_{\mathbf{Z}_{2}},
\end{array}
$$

we see that they get carried to the $M O^{*}$-multiples of $\beta_{1}^{-1}$ down the left side. Anticipating a calculation we will carry out in detail below, each summand of $H \Phi$ corresponding to one of these multiples of $\beta_{1}^{-1}$ is carried into a single 
summand of $E \mathbf{Z}_{2}^{+} \wedge H \Omega$, corresponding to the same coefficient. This, by the "probing" idea above, is sufficient to show that each summand of $E \mathbf{Z}_{2}^{+} \wedge H \Omega$ is carried into itself, as we claimed.

But then we have for each summand a map $E \mathbf{Z}_{2}^{+} \wedge H \Omega \rightarrow \Sigma^{L-1} E \mathbf{Z}_{2}^{+} \wedge H \Omega$ which is an equivalence; since there is only one nontrivial map of this sort, by Theorem 2.13, this map must be $\theta$.

Thus $\widetilde{E Z}_{2} \wedge M O_{\mathbf{Z}_{2}}$ is already a wedge of Eilenberg-Mac Lane spectra, but $E \mathrm{Z}_{2}^{+} \wedge M \mathrm{O}_{\mathrm{Z}_{2}}$ is not. We need to make some modifications in order to make the fibration sequence into a Postnikov tower.

First we examine the connecting map $\widetilde{E \mathbf{Z}}_{2} \wedge M O_{\mathbf{Z}_{2}} \rightarrow \Sigma E \mathbf{Z}_{2}^{+} \wedge M O_{\mathbf{Z}_{2}}$. Following the proof of Corollary 2.11, we compute the map $\left[H \Phi, \widetilde{E \mathbf{Z}_{2}} \wedge M O_{\mathbf{Z}_{2}}\right]_{\mathbf{Z}_{2}}^{*} \rightarrow$ $\left[H \Phi, \Sigma E \mathbf{Z}_{2}^{+} \wedge M O_{\mathbf{Z}_{2}}\right]_{\mathbf{Z}_{2}}^{*}$. Recalling that $H \Phi \simeq \widetilde{E \mathbf{Z}}_{2} \wedge p^{*} H \mathbf{Z}_{2}$ and $\widetilde{E Z}_{2}=$ $\operatorname{colim}_{b} S^{b L}$, we approximate $H \Phi$ with the spectra $\Sigma^{b L} p^{*} H Z_{2}$. More generally, consider the spectra of the form $\Sigma^{b L} p^{*} A$, where $A$ is any nonequivariant spectrum. Using the fact that $\left[\Sigma^{b L} p^{*} A, Y\right]_{\mathrm{Z}_{2}}^{*} \cong\left[p^{*} A, \Sigma^{-b L} Y\right]_{\mathrm{Z}_{2}}^{*}$ we are back to studying cohomology theories on nonequivariant spectra. Thus we look at $\left[p^{*} A, \Sigma^{-b L} \widetilde{E \mathbf{Z}}_{2} \wedge M O_{\mathbf{Z}_{2}}\right]_{\mathbf{Z}_{2}}^{*} \rightarrow\left[p^{*} A, \Sigma^{-b L} \Sigma E \mathbf{Z}_{2}^{+} \wedge M O_{\mathbf{Z}_{2}}\right]_{\mathbf{Z}_{2}}^{*}$. Now

$$
\left(\Sigma^{-b L} \widetilde{E \mathbf{Z}}_{2} \wedge M O_{\mathbf{Z}_{2}}\right)^{*} \cong \Sigma^{b} \underline{\mathscr{M}}^{*},
$$

using the fact that $\Sigma^{-b L} M O_{\mathrm{Z}_{2}} \simeq \Sigma^{-b} M O_{\mathrm{z}_{2}}$. We will write $\Sigma^{b} x$ for the element in dimension $|x|+b$ of $\Sigma^{b} \mathscr{M}^{*}$ corresponding to $x$ in $\mathscr{M}^{*}$. Similarly, $\left(\Sigma^{-b L} \Sigma E \mathbf{Z}_{2}^{+} \wedge M O_{\mathbf{Z}_{2}}\right)^{*} \cong \Sigma^{b-1} M O^{*}\left\{r_{0}, r_{1}, \ldots\right\}$. By abuse of notation, we will write $r_{i-b+1}$ for $\Sigma^{b-1} r_{i}$. Thus

$$
\left(\Sigma^{-b L} \Sigma E \mathbf{Z}_{2}^{+} \wedge M O_{\mathbf{Z}_{2}}\right)^{*} \cong M O^{*}\left\{r_{-b+1}, r_{-b+2}, \ldots\right\} .
$$

We apply Corollary 1.10 to get

Lemma 3.4. If $A$ is a nonequivariant spectrum, then

$$
\left(\widetilde{E \mathbf{Z}}_{2} \wedge M O_{\mathbf{Z}_{2}}\right)^{*}\left(\Sigma^{b L} p^{*} A\right) \cong M O^{*}(A) \widehat{\otimes}_{M O^{*}} \Sigma^{b} \underline{\mathscr{M}}^{*}
$$

and

$$
\left(E \mathbf{Z}_{2}^{+} \wedge M O_{\mathbf{Z}_{2}}\right)^{*}\left(\Sigma^{b L} p^{*} A\right) \cong M O^{*}(A) \widehat{\otimes}_{M O^{*}} M O^{*}\left\{r_{-b+1}, r_{-b+2}, \ldots\right\} .
$$

Moreover, the following diagram commutes:

$$
\begin{aligned}
& M O^{*}(A) \hat{\otimes} \Sigma^{b} \mathscr{M}^{*} \cong\left(\widetilde{E Z}_{2} \wedge M O_{\mathbf{Z}_{2}}\right)^{*}\left(\Sigma^{b L} p^{*} A\right) \\
& M O^{*}(A) \hat{\otimes} \Sigma^{b-1} M O^{*}\left\{r_{0}, r_{2}, \ldots\right\} \cong\left(\Sigma E \mathbf{Z}_{2}^{+} \wedge M O_{\mathbf{Z}_{2}}\right)^{*}\left(\Sigma^{b L} p^{*} A\right) .
\end{aligned}
$$

(Note: the tensor products above are completed tensor products.) 
Thus we need to study $\Sigma^{b} \partial$. As $b$ varies, these maps are related by

$$
\begin{aligned}
\Sigma^{b} \stackrel{\mathscr{M}^{*}}{\stackrel{\Sigma^{b} \partial}{\longrightarrow}} M O^{*}\left\{r_{-b+1}, r_{-b+2}, \ldots\right\} \\
\downarrow \beta_{1}^{-1} \\
\Sigma^{b-1} \stackrel{\mathscr{M}^{*}}{\stackrel{\Sigma^{b-1} \partial}{\longrightarrow}} M O^{*}\left\{r_{-b+2}, r_{-b+3}, \ldots\right\}
\end{aligned}
$$

where the map labeled $\beta_{1}^{-1}$ is given by $\Sigma^{b} x \mapsto \Sigma^{b-1}\left(\beta_{1}^{-1} x\right)$, and the map on the right is given by $r_{k} \mapsto r_{k}$ for $k>-b+1$, and $r_{-b+1}$ maps to 0 . These are the maps induced by the inclusion $S^{(b-1) L} \rightarrow S^{b L}$, since $\beta_{1}^{-1}$ is the Euler class of the representation $L$, and we can see by representing the $r_{i}$ as explicit free $\mathbf{Z}_{2}$-manifolds that $\beta_{1}^{-1}$ acts on them to produce exactly this map.

Write $(s)_{k}$ for the coefficient of $r_{k}$ in $s \in M O^{*}\left\{r_{-b+1}, \ldots\right\}$. Then we know that $\left(\Sigma^{b} \partial\left(\Sigma^{b} x\right)\right)_{k}=(\partial x)_{k+b}$ for $k>-b$, by definition, and also $\left(\Sigma^{b} \partial\left(\Sigma^{b} x\right)\right)_{k}$ $=\left(\partial \beta_{1}^{-b} x\right)_{k}$ for $k \geq 1$, by the diagram above. Thus $(\partial x)_{k+b}=\left(\partial \beta_{1}^{-b} x\right)_{k}$ for $k \geq 1$.

We now take the limit over $b$. Since $\beta_{1}^{-1}$ has an inverse, namely $\beta_{1}$, we have $\Sigma^{b} \mathscr{M}^{*} \cong \mathscr{M}^{*}$ by $\Sigma^{b} x \mapsto \beta_{1}^{-b} x$. So $\lim _{b} \Sigma^{b} \underline{\mathscr{M}}^{*} \cong \mathscr{M}^{*}$. On the other hand, we can see that

$$
\lim _{b} M O^{*}\left\{r_{-b+1}, \ldots\right\} \cong M O^{*}\left\{\left\{r_{i} \mid i \in \mathbf{Z}\right\}\right\},
$$

where the double braces indicate the module of infinite homogeneous linear combinations of the elements $r_{i}$. Let us write this last module as $M O^{*}\left[\left[\theta, \theta^{-1}\right]\right]$, where $|\theta|=-1$, and think of elements of this module as Laurent series in $\theta$, with only finitely many terms of positive degree in $\theta$. We are justified in reusing the letter $\theta$ here by Corollary 3.3.

Write $\Sigma^{\infty} \partial$ for the limit of the maps $\Sigma^{b} \partial$. We see then that

$$
\begin{aligned}
\Sigma^{\infty} \partial(x) & =\sum_{k=-\infty}^{\infty}\left(\Sigma^{-k+1} \partial\left(\Sigma^{-k+1} \beta_{1}^{-k+1} x\right)\right)_{k} \theta^{k} \\
& =\sum_{k=-\infty}^{\infty}\left(\partial\left(\beta_{1}^{-k+1} x\right)\right)_{1} \theta^{k} \\
& =\sum_{k=0}^{\infty}\left(\partial\left(\beta_{1}^{k+1} x\right)\right)_{1} \theta^{-k}+\sum_{k=1}^{\infty}(\partial x)_{k} \theta^{k} .
\end{aligned}
$$

We can describe the coefficients in this series more geometrically. The map

$$
\imath: M O^{*}\left\{r_{1}, r_{2}, \ldots\right\} \rightarrow \underline{\mathscr{M}}^{*}
$$

given by $l\left(r_{i}\right)=\beta_{1}^{i}$ is an explicit splitting of the short exact sequence of Theorem 3.1 (writing, as usual, $r_{i}$ for $\Sigma^{-1} r_{i-1}$ ), showing that $\mathscr{M}^{*} \cong M O_{\mathbf{Z}_{2}}^{*} \oplus$ $M O^{*}\left\{r_{1}, r_{2}, \ldots\right\}$ as $M O^{*}$-modules. $\partial x$ can then be described as follows: since $x+l \partial x \in M O_{\mathbf{Z}_{2}}^{*}$, the coefficients of $\partial x$ are the (unique) elements of $M O^{*}$ 
such that $x+\Sigma_{k=1}^{\infty}(\partial x)_{k} \beta_{1}^{k} \in M O_{\mathbf{Z}_{2}}^{*}$. Now recall the map $\Gamma: M O_{\mathbf{Z}_{2}}^{*} \rightarrow M O_{\mathbf{Z}_{2}}^{*-1}$ from [2]. It is given by the formula $\Gamma(x)=\beta_{1} x+\varepsilon(x) \beta_{1}$, if we work in $\mathscr{M}^{*}$, where $\varepsilon: M O_{\mathrm{Z}_{2}}^{*} \rightarrow M O^{*}$ is the forgetful map. Therefore, if $x \in M O_{\mathbf{Z}_{2}}^{*}$, $\partial\left(\beta_{1} x\right)=\varepsilon(x) r_{1}$. By induction, we can show, for any $x \in M O_{\mathbf{Z}_{2}}^{*}$,

$$
\partial\left(\beta_{1}^{k+1} x\right)_{1}=\varepsilon \Gamma^{k}(x), \quad k \geq 0
$$

and for any $x \in \underline{\mathscr{M}}^{*}$,

$$
\partial\left(\beta_{1}^{k+1} x\right)_{1}=\varepsilon \Gamma^{k}(x+\imath \partial x), \quad k \geq 0 .
$$

This gives another form for the coefficients of $\Sigma^{\infty} \partial x$, i.e.,

$$
\Sigma^{\infty} \partial x=\sum_{k=0}^{\infty} \varepsilon \Gamma^{k}(x+\imath \partial x) \theta^{-k}+\sum_{k=1}^{\infty}(\partial x)_{k} \theta^{k} .
$$

In this form, we can see that $\Sigma^{\infty} \partial$ is essentially the map $J$ studied by Boardman in [3]. His results show that, for $x$ not a polynomial in $\beta_{1}$ and $\beta_{1}^{-1}$, infinitely many of these coefficients are nonzero, and that $\Sigma^{\infty} \partial$ is an injective map.

Since, among other things, these results give that the inverse limit systems over $b$ consist of surjective maps, we have

Corollary 3.5. If $A$ is a nonequivariant spectrum, then we have

$$
\begin{aligned}
& M O^{*}(A) \widehat{\otimes}_{M O^{*}} \mathscr{\mathscr { M }}^{*} \cong\left(\widetilde{E \mathbf{Z}}_{2} \wedge M O_{\mathbf{Z}_{2}}\right)^{*}\left(\widetilde{E \mathbf{Z}}_{2} \wedge p^{*} A\right) \\
& M O^{*}(A) \widehat{\otimes}_{M O^{*}} M O^{*}\left[\left[\theta, \theta^{-1}\right]\right] \cong\left(\Sigma E \mathbf{Z}_{2}^{+} \wedge M O_{\mathbf{Z}_{2}}\right)^{*}\left(\widetilde{E \mathbf{Z}}_{2} \wedge p^{*} A\right) \text {. }
\end{aligned}
$$

In particular, letting $A=\mathrm{HZ}_{2}$, we have

Corollary 3.6. The following diagram commutes:

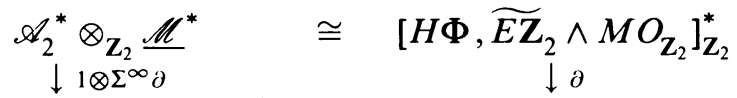

$$
\begin{aligned}
& \mathscr{A}_{2}^{*} \otimes_{\mathrm{Z}_{2}} M O^{*}\left[\left[\theta, \theta^{-1}\right] \cong\left[H \Phi, \Sigma E \mathbf{Z}_{2}^{+} \wedge M O_{\mathbf{Z}_{2}}\right]_{\mathrm{Z}_{2}}^{*}\right. \text {. }
\end{aligned}
$$

Thus $\partial \in\left[\widetilde{E Z}_{2} \wedge M O_{\mathbf{Z}_{2}}, \Sigma E \mathbf{Z}_{2}^{+} \wedge M O_{\mathbf{Z}_{2}}\right]_{\mathbf{Z}_{2}} \cong \operatorname{Hom}_{\mathbf{Z}_{2}}\left(\mathscr{M}^{*}, \mathscr{A}_{2}^{*} \widehat{\otimes} M O^{*}\left[\left[\theta, \theta^{-1}\right]\right)\right.$ by Corollary 2.11 , is given by $\Sigma^{\infty} \partial$, which is given by the formulae above.

Corollary 3.7. Any composite $H \Phi \rightarrow M O_{\mathbf{z}_{2}} \rightarrow H \Phi$ is $\mathrm{Z}_{2}$-trivial.

Proof. Since $\Sigma^{\infty} \partial$ is injective, any composite $H \Phi \rightarrow M O_{\mathbf{Z}_{2}} \rightarrow \widetilde{E \mathbf{Z}_{2}} \wedge M O_{\mathbf{Z}_{2}}$ is trivial by Corollary 3.6. But by Theorems 1.2 and 2.1, any map $M O_{\mathrm{Z}_{2}} \rightarrow H \Phi$ factors through $\widetilde{E Z}_{2} \wedge M O_{\mathrm{Z}_{2}}$, so the result follows.

Thus $\mathrm{MO}_{\mathrm{Z}}$ splits off no factors looking like $H \Phi$, dashing any hopes that it is a wedge of Eilenberg-Mac Lane spectra.

Now we want to convert the fibration that we started with into an honest Postnikov tower. Taking the splitting $\underline{\mathscr{M}}^{*} \cong M O_{\mathrm{Z}_{2}}^{*} \otimes M O^{*}\left\{\beta_{1}, \beta_{1}^{2}, \ldots\right\}$ induced by $l$, we can write

$$
\widetilde{E Z}_{2} \wedge M O_{\mathrm{Z}_{2}} \simeq H\left(\Phi \otimes \underline{\mathscr{M}}^{*}\right) \simeq H\left(\Phi \otimes M O_{\mathrm{Z}_{2}}^{*}\right) \vee H\left(\Phi \otimes M O^{*}\left\{\beta_{1}, \beta_{1}^{2}, \ldots\right\}\right)
$$


Notice that the map $H\left(\Phi \otimes \mathscr{M}^{*}\right) \rightarrow H\left(\Phi \otimes M O_{\mathrm{Z}_{2}}^{*}\right)$ is the one induced by $\underline{\mathscr{M}}^{*} \rightarrow M O_{\mathrm{Z}_{2}}^{*}$, the latter map being given by $x \mapsto x+\imath \partial x$.

Recall that Corollary 3.3 says that $E \mathbf{Z}_{2}^{+} \wedge M O_{\mathbf{Z}_{2}} \simeq E \mathbf{Z}_{2}^{+} \wedge H\left(\Omega \otimes M O^{*}\right)$. Therefore there is a fibration sequence $\widetilde{E Z}_{2} \wedge H\left(\Omega \otimes M O^{*}\right) \rightarrow \Sigma E \mathrm{Z}_{2}^{+} \wedge M O_{\mathrm{Z}_{2}} \rightarrow$ $\Sigma H\left(\Omega \otimes M O^{*}\right)$. Further, we know by Theorem 2.6 that $\widetilde{E Z}_{2} \wedge H\left(\Omega \otimes M O^{*}\right) \simeq$ $H\left(\Phi \otimes M O^{*}\left\{\beta_{1}, \beta_{1}^{2}, \ldots\right\}\right)$. By Theorem 2.7, these two maps into $\Sigma E \mathbf{Z}_{2}^{+} \wedge M O_{\mathbf{Z}_{2}}$ from $H\left(\Phi \otimes M O^{*}\left\{\beta_{1}, \beta_{1}^{2}, \ldots\right\}\right)$ differ at most by a self-equivalence of the latter spectrum. Thus we can fill in the rightmost vertical arrow in

$$
\begin{aligned}
& H\left(\Phi \otimes M O^{*}\left\{\beta_{1}, \beta_{1}^{2}, \ldots\right\}\right) \rightarrow H\left(\Phi \otimes \underline{\mathscr{M}^{*}}\right) \rightarrow H\left(\Phi \otimes M O_{\mathrm{Z}_{2}}^{*}\right) \\
& \downarrow \simeq \\
& H\left(\Phi \otimes M O^{*}\left\{\beta_{1}, \beta_{1}^{2}, \ldots\right\}\right) \rightarrow \Sigma E \mathbf{Z}_{2}^{+} \wedge M O_{\mathbf{Z}_{2}} \rightarrow \Sigma H\left(\Omega \otimes M O^{*}\right) .
\end{aligned}
$$

This implies that we can replace our original fibration with the fibration sequence

$$
H\left(\Omega \otimes M O^{*}\right) \rightarrow M O_{\mathrm{z}_{2}} \rightarrow H\left(\Phi \otimes M O_{\mathrm{z}_{2}}^{*}\right) .
$$

Putting this all together, we have

Theorem 3.8. $M O_{\mathrm{Z}_{2}}$ is the fiber of the map $H\left(\Phi \otimes M O_{\mathrm{Z}_{2}}^{*}\right) \rightarrow \Sigma H\left(\Omega \otimes M O^{*}\right)$ corresponding to the homomorphism $\Sigma^{\infty} \partial \in \operatorname{Hom}_{\mathrm{Z}_{2}}\left(M O_{\mathrm{Z}_{2}}^{*}, \mathscr{A}_{2}^{*} \widehat{\otimes} M O^{*}\left[\left[\theta^{-1}\right]\right]\right)$, where $\Sigma^{\infty} \partial x=\sum_{k=0}^{\infty} \varepsilon \Gamma^{k}(x) \theta^{-k}$.

Although these results, together with Boardman's [3], show that $M O_{\mathrm{z}_{2}}$ is not a wedge of Eilenberg-Mac Lane spectra, they do give us one interesting splitting. Corollary 3.9. $M O_{\mathrm{Z}_{2}} \simeq T \vee \widehat{M O}_{\mathrm{Z}_{2}}$ for some $\mathrm{Z}_{2}$-spectrum $\widehat{M O}_{\mathrm{Z}_{2}}$, where $T$ is the fiber of the map $t: H\left(\Phi \otimes \mathbf{Z}_{2}\left[\beta_{1}^{-1}\right]\right) \rightarrow \Sigma H \Omega$ corresponding to the homomorphism $\mathbf{Z}_{2}\left[\beta_{1}^{-1}\right] \rightarrow \mathscr{A}_{2}^{*}\left[\theta^{-1}\right]$ given by $\beta_{1}^{-k} \mapsto \theta^{-k}$.

Proof. This follows from an algebraic splitting. Write $M O^{*}=\mathrm{Z}_{2} \oplus \widehat{M O}^{*}$ where $\widehat{M O}^{*}$ is the set of elements of $M O^{*}$ of strictly negative degrees. Then we can write $M O^{*}\left[\left[\theta^{-1}\right]\right]=\mathbf{Z}_{2}\left[\theta^{-1}\right] \oplus \widehat{M O}^{*}\left[\left[\theta^{-1}\right]\right]$.

Now $M O_{\mathbf{Z}_{2}}^{*}$ has a subalgebra $\mathrm{Z}_{2}\left[\beta_{1}^{-1}\right]$. The map $\Sigma^{\infty} \partial$ carries this subalgebra isomorphically onto the subalgebra $\mathrm{Z}_{2}\left[\theta^{-1}\right]$ of $M O^{*}\left[\left[\theta^{-1}\right]\right]$. Let $\widehat{M O}_{\mathrm{Z}_{2}}^{*}$ be the kernel of the composite $M O_{\mathrm{Z}_{2}}^{*} \rightarrow M O^{*}\left[\left[\theta^{-1}\right]\right] \rightarrow \mathrm{Z}_{2}\left[\theta^{-1}\right]$. Then $\Sigma^{\infty} \partial$ carries $\widehat{M O}_{\mathrm{Z}_{2}}^{*}$ into $\widehat{M O}^{*}\left[\left[\theta^{-1}\right]\right]$. Thus we can write $\Sigma^{\infty} \partial$ as the direct sum of the two maps $\mathbf{Z}_{2}\left[\beta_{1}^{-1}\right] \rightarrow \mathbf{Z}_{2}\left[\theta^{-1}\right]$ and $\widehat{M O}_{\mathbf{Z}_{2}}^{*} \rightarrow \widehat{M O}^{*}\left[\left[\theta^{-1}\right]\right]$. This algebraic splitting gives rise to the splitting of the spectrum $M O_{\mathrm{Z}_{2}}$.

We will use the spectrum $T$ to study transfers and cohomology operations in $\S 5$.

\section{The STRUCTURE OF $m O_{\mathbf{z}_{2}}$}

Although $\mathrm{MO}_{\mathrm{Z}_{2}}$ does not represent geometric $\mathrm{Z}_{2}$-bordism, there is a spectrum which does, which we will call $\mathrm{mO}_{\mathrm{z}_{2}}$. It can be defined explicitly in the 
following way. If $V$ is a real representation of $\mathrm{Z}_{2}$, let $b O_{\mathrm{Z}_{2}}(V)$ be the space of $|V|$-dimensional planes in $V \oplus \mathbf{R}^{\infty}$, with $\mathbf{Z}_{2}$ acting by translation of planes ( $\mathbf{R}$ denotes the trivial one-dimensional representation of $\mathbf{Z}_{2}$ ). This has an obvious $|V|$-dimensional $\mathbf{Z}_{2}$-vector bundle over it, and we let $m O_{\mathbf{z}_{2}}(V)$ be the Thom space of this bundle. (Following [10], we should say that this defines a prespectrum, and let $m \mathrm{O}_{\mathrm{Z}_{2}}$ denote the corresponding spectrum.) It is well known that this spectrum represents geometric $\mathbf{Z}_{2}$-bordism; see, for example $\left[14,13\right.$, or 4]. In conformity with our previous notation, we write $m \mathrm{O}_{\mathrm{z}_{2}}^{*}$ for the geometric bordism groups, graded cohomologically. It is obvious that there is a $\mathrm{Z}_{2}$-map of spectra $m O_{\mathrm{Z}_{2}} \rightarrow M O_{\mathrm{Z}_{2}}$ which induces the obvious map from geometric bordism to stable bordism.

Although we know that $m O_{\mathrm{z}_{2}}$ exists, an interesting question is: In what sense is it unique? We cannot appeal to a $Z_{2}$-version of Corollary 1.8 , since geometric bordism is only naturally defined in integer grading. The problem is exactly the one pointed out in Theorem 2.12. As we will see, we can appeal to some property of $\mathrm{mO}_{\mathrm{z}_{2}}$ beyond the fact that it represents geometric bordism to get its uniqueness. We will get one such uniqueness result in this section, and another in the next.

Suppose then that $X$ is any $\mathbf{Z}_{2}$-spectrum which naturally represents geometric $\mathbf{Z}_{2}$-bordism, meaning that for any $\mathbf{Z}_{2}$-space $A, \tilde{X}_{*}(A)$ is naturally isomorphic, as $\mathbf{Z}$-graded homology theory on $\mathbf{Z}_{2}$-spaces, to the $\mathbf{Z}$-graded $\mathbf{Z}_{2}$ homology theory given by the geometric $\mathrm{Z}_{2}$-bordism groups of $A$. Let $\mathscr{M}^{*}=$ $M O^{*}\left[\beta_{1}, \beta_{2}, \ldots\right]$ where $\left|\beta_{i}\right|=-i$. Again, we have a short exact sequence of free $M O^{*}$-modules,

$$
0 \rightarrow m O_{\mathbf{Z}_{2}}^{*} \rightarrow \mathscr{M}^{*} \rightarrow M O^{*}\left\{r_{1}, r_{2}, \ldots\right\} \rightarrow 0,
$$

as in [2], and $\left(\widetilde{E \mathbf{Z}}_{2} \wedge X\right)^{*} \cong \mathscr{M}^{*}$. As in $\S 3$ we can show

Lemma 4.1. If $X$ naturally represents geometric $\mathbf{Z}_{2}$-bordism, then $\widetilde{E Z}_{2} \wedge X \simeq$ $H\left(\Phi \otimes \mathscr{M}^{*}\right)$.

Also, since we do have $i^{*} X \simeq M O$ by Corollary 1.8 , and, as in $\S 3, X$ must be split, we get

Lemma 4.2. If $X$ naturally represents geometric $\mathbf{Z}_{2}$-bordism, then $E \mathbf{Z}_{2}^{+} \wedge X \simeq$ $E \mathbf{Z}_{2}^{+} \wedge H\left(\Omega \otimes M O^{*}\right)$.

Now consider the connecting map $\widetilde{E Z}{ }_{2} \wedge X \rightarrow \Sigma E \mathbf{Z}_{2}^{+} \wedge X$, or $H\left(\Phi \otimes \mathscr{M}^{*}\right) \rightarrow$ $\Sigma E \mathbf{Z}_{2}^{+} \wedge H\left(\Omega \otimes M O^{*}\right)$. There is a submodule $M O^{*}\left\{\beta_{1}, \beta_{1}^{2}, \ldots\right\} \subset \mathscr{M}^{*}$ and $\mathscr{M}^{*} \cong M O^{*}\left\{\beta_{1}, \beta_{1}^{2}, \ldots\right\} \oplus m O_{\mathbf{Z}_{2}}^{*}$. Further, the restriction of the connecting map to

$$
H\left(\Phi \otimes M O^{*}\left\{\beta_{1}, \beta_{1}^{2}, \ldots\right\}\right) \rightarrow \Sigma E \mathbf{Z}_{2}^{+} \wedge H\left(\Omega \otimes M O^{*}\right)
$$

is a homotopy isomorphism on fixed-points. Thus, as in the previous section, 
we use Theorem 2.7 to conclude

Theorem 4.3. If $X$ is a $\mathbf{Z}_{2}$-spectrum which naturally represents geometric $\mathbf{Z}_{2}$ bordism on spaces, then there is a fibration

$$
X \rightarrow H\left(\Phi \otimes m O_{\mathrm{Z}_{2}}^{*}\right) \rightarrow \Sigma H\left(\Omega \otimes M O^{*}\right) .
$$

The problem now is to determine the $k$-invariant in this two-stage Postnikov tower. Without further hypotheses on $X$, however, there is little that we can say. In fact, Theorem 2.12 and the existence of $\mathrm{mO}_{\mathrm{z}_{2}}$ give us this converse of Theorem 4.3:

Corollary 4.4. Suppose that $X$ is the fiber of any map from $H\left(\Phi \otimes m O_{*}^{\mathbf{z}_{2}}\right)$ to $\Sigma H\left(\Omega \otimes M O_{*}\right)$. Then if $A$ is a $\mathbf{Z}_{2}-C W$-complex, there is a natural isomorphism

$$
\tilde{X}_{*}(A) \cong \widetilde{M O_{*}}\left(\left(A / \mathbf{Z}_{2}\right) / A^{\mathbf{Z}_{2}}\right) \oplus\left(\widetilde{M O_{*}}\left(A^{\mathbf{Z}_{2}}\right) \otimes_{M O_{*}} m O_{*}^{\mathbf{Z}_{2}}\right),
$$

and therefore a natural isomorphism $\tilde{X}_{*}(A) \cong \widetilde{m O}_{*}^{\mathbf{Z}_{2}}(A)$. Thus any such $X$ naturally represents geometric $\mathbf{Z}_{2}$-bordism on spaces.

What, then, distinguishes $\mathrm{mO}_{\mathrm{z}_{2}}$ from all of these other spectra? Here is our first answer, together with the structure of $m \mathrm{O}_{\mathrm{z}_{2}}$ :

Theorem 4.5. If $X$ is a $\mathbf{Z}_{2}$-spectrum which naturally represents geometric $\mathbf{Z}_{2}$ bordism, and if there is a $\mathbf{Z}_{2}$-map $X \rightarrow M O_{\mathbf{Z}_{2}}$ which represents the obvious transformation from geometric to stable bordism, then $X$ is equivalent to $m O_{\mathrm{Z}_{2}}$. Moreover, $m \mathrm{O}_{\mathrm{z}_{2}}$ is the fiber of the particular map $H\left(\Phi \otimes m O_{\mathrm{Z}_{2}}^{*}\right) \rightarrow \Sigma H\left(\Omega \otimes M O^{*}\right)$ corresponding to

$$
\Sigma^{\infty} \partial: m O_{\mathrm{Z}_{2}}^{*} \rightarrow \mathscr{A}_{2}^{*} \widehat{\otimes} M O^{*}\left[\left[\theta^{-1}\right]\right]
$$

where $\Sigma^{\infty} \partial x=\sum_{k=0}^{\infty} \varepsilon \Gamma^{k}(x) \theta^{-k}$

Proof. If $X$ has the properties stated, then the map $X \rightarrow M O_{\mathrm{z}_{2}}$ gives a diagram

$$
\begin{array}{ccccc}
H\left(\Omega \otimes M O^{*}\right) & \rightarrow & X & \rightarrow & H\left(\Phi \otimes m O_{\mathbf{Z}_{2}}^{*}\right) \\
\downarrow= & & \downarrow & & \downarrow \\
H\left(\Omega \otimes M O^{*}\right) & \rightarrow & M O_{\mathbf{Z}_{2}} & \rightarrow & H\left(\Phi \otimes M O_{\mathbf{Z}_{2}}^{*}\right)
\end{array}
$$

where the rightmost vertical arrow is induced by the inclusion $m O_{\mathrm{Z}_{2}}^{*} \rightarrow M O_{\mathrm{Z}_{2}}^{*}$. This we can see by examining how we constructed the top row. From this and Theorem 3.8 we can read off the $k$-invariant for $X$ : it is given by $\Sigma^{\infty} \partial$. On the other hand, the diagram above clearly determines $X$ up to equivalence; since $m \mathrm{O}_{\mathrm{z}_{2}}$ is known to satisfy the hypotheses, all such $X$ must be equivalent to $m O_{\mathrm{Z}_{2}}$, and $m O_{\mathrm{Z}_{2}}$ has the stated structure.

Again, we can deduce that, although $\mathrm{mO}_{\mathrm{z}_{2}}$ is not a wedge of EilenbergMac Lane spectra, there is some splitting.

Corollary 4.6. $m O_{\mathrm{Z}_{2}} \simeq H \underline{\mathrm{Z}}_{2} \vee \widehat{m O}_{\mathrm{Z}_{2}}$ for some spectrum $\widehat{m O}_{\mathrm{Z}_{2}}$.

Proof. The proof is essentially the same as that of Corollary 3.9. In this case, though, we notice that one factor looks like the fiber of the unique nontrivial 
map $H \Phi \rightarrow \Sigma H \Omega$, and we know that that fiber is $H \underline{\mathrm{Z}}_{2}$, from the short exact sequence of Mackey functors

$$
0 \rightarrow \Omega \rightarrow \underline{\mathbf{Z}}_{2} \rightarrow \Phi \rightarrow 0
$$

\section{TRANSFERS}

The uniqueness result proved at the end of the last section is unsatisfactory, in the sense that the existence of a map into $M O_{\mathrm{Z}_{2}}$ is a somewhat unnatural extrinsic condition. We would like a more intrinsic condition, and in this section we will find one in terms of transfers in the bordism homology theory.

We start by studying equivariant transfers in the homology theories represented by spectra given by two-stage Postnikov towers of the sort we have been studying. The transfers we are interested in are somewhat special: they are the transfers associated with double covering maps of the form $A \rightarrow A / \mathbf{Z}_{2}$, where $A$ is a free $\mathbf{Z}_{2}$-space (and we consider $A / \mathbf{Z}_{2}$ to be a trivial $\mathbf{Z}_{2}$-space). Associated to such a map is the stable transfer map $\Sigma^{\infty} A / Z_{2} \rightarrow \Sigma^{\infty} A$, which induces, for any $\mathbf{Z}_{2}$-spectrum $X$, a map $\tau: X_{*}\left(A / \mathbf{Z}_{2}\right) \rightarrow X_{*}(A)$ [10]. Our first object then is to study $\tau$ for our special spectra $X$.

Before doing that, let us remark that we know geometrically what this transfer looks like in the bordism theories. If $M \rightarrow A / \mathbf{Z}_{2}$ is a $\mathbf{Z}_{2}$-manifold over $A / \mathbf{Z}_{2}$, then $\tau$ carries it to the class of the manifold $\widetilde{M}$ over $A$, where $\widetilde{M}$ is the pullback in the diagram

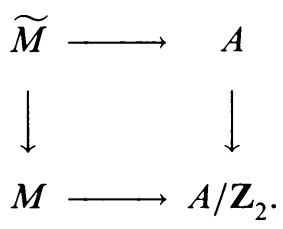

This is the standard description of the transfer in bordism theories. To check that this agrees with the map $\tau$ is an easy exercise using the Pontrjagin-Thom construction that shows how bordism theories are represented by Thom spectra in the first place.

Consider the $\mathrm{Z}_{2}$-spectrum $T$ that we split off from $M O_{\mathrm{Z}_{2}}$ in $\S 3$. Recall that it is the fiber of the map $H\left(\Phi \otimes \mathbf{Z}_{2}\left[\beta_{1}^{-1}\right]\right) \rightarrow \Sigma H \Omega$ specified in the usual way by $\beta_{1}^{-k} \mapsto \theta^{-k}$.

Theorem 5.1. If $A$ is a free $\mathbf{Z}_{2}-C W$-complex, then

$$
T_{*}(A) \cong H_{*}^{\mathbf{Z}_{2}}(A ; \Omega) \cong H_{*}\left(A / \mathbf{Z}_{2}\right),
$$

and

$$
\begin{aligned}
T_{*}\left(A / \mathbf{Z}_{2}\right) & \cong H_{*}^{\mathbf{Z}_{2}}\left(A / \mathbf{Z}_{2} ; \Phi\right) \otimes_{\mathbf{Z}_{2}} \mathbf{Z}_{2}\left[\beta_{1}^{-1}\right] \\
& \cong H_{*}\left(A / \mathbf{Z}_{2}\right) \otimes_{\mathbf{Z}_{2}} \mathbf{Z}_{2}\left[\beta_{1}^{-1}\right]
\end{aligned}
$$


where the homological degree of $\beta_{1}^{-1}$ is -1 . The transfer $\tau: H_{*}\left(A / \mathbf{Z}_{2}\right) \otimes_{\mathbf{Z}_{2}}$ $\mathbf{Z}_{2}\left[\beta_{1}^{-1}\right] \rightarrow H_{*}\left(A / \mathbf{Z}_{2}\right)$ is given by

$$
\tau\left(x \otimes \beta_{1}^{-k}\right)=w_{1}^{k} \cap x
$$

where $w_{1} \in H^{1}\left(A / \mathbf{Z}_{2}\right)$ is the first Stiefel-Whitney class of the bundle $A \rightarrow A / \mathbf{Z}_{2}$. Proof. The calculations of $T_{*}(A)$ and $T_{*}\left(A / \mathbf{Z}_{2}\right)$ follow when we notice that $H_{*}^{\mathbf{Z}_{2}}(A ; \Phi)=0$ because $A$ is free, and $H_{*}^{\mathbf{Z}_{2}}\left(A / \mathbf{Z}_{2} ; \Omega\right)=0$ because $\mathbf{Z}_{2}$ acts trivially on $A / \mathbf{Z}_{2}$.

To find the transfer, consider the projection $M O_{*}^{\mathbf{Z}_{2}}(B) \rightarrow T_{*}(B)$ for any space $B$, given by the fact that $T$ is a wedge summand of $M O_{\mathrm{z}_{2}}$. Notice that $M O_{*}^{\mathbf{Z}_{2}}\left(A / \mathbf{Z}_{2}\right) \cong M O_{*}\left(A / \mathbf{Z}_{2}\right) \otimes_{M O *} M O_{*}^{\mathbf{Z}_{2}}$ by Theorem 3.8, and the summand

$$
M O_{*}\left(A / \mathbf{Z}_{2}\right) \otimes_{M O_{*}} M O_{*}\left[\beta_{1}^{-1}\right] \cong M O_{*}\left(A / \mathbf{Z}_{2}\right) \otimes_{\mathbf{Z}_{2}} \mathbf{Z}_{2}\left[\beta_{1}^{-1}\right]
$$

maps onto $T_{*}\left(A / \mathbf{Z}_{2}\right)$ by means of the usual projection

$$
M O_{*}\left(A / \mathbf{Z}_{2}\right) \rightarrow H_{*}\left(A / \mathbf{Z}_{2}\right) .
$$

If $x \otimes \beta_{1}^{-k} \in T_{*}\left(A / \mathbf{Z}_{2}\right)$ then, there exists a $\mathbf{Z}_{2}$-trivial manifold $M$ over $A / \mathbf{Z}_{2}$ such that $x \otimes \beta_{1}^{-k}$ is the image of the stable manifold $M \rightarrow D\left(L^{k}\right)$ over $A / \mathbf{Z}_{2}$, where $M$ maps entirely into the origin of $D\left(L^{k}\right)$. (It can be shown that $\beta_{1}^{-k}$ is represented by the stable manifold given by inclusion of the origin in $D\left(L^{k}\right)$.) Write $m \in M O_{*}^{\mathbf{Z}_{2}}\left(A / \mathbf{Z}_{2}\right)$ for the class of $M \rightarrow D\left(L^{k}\right)$. Then, as noted above, $\tau(m)$ is represented by the stable manifold $\widetilde{M} \rightarrow D\left(L^{k}\right)$, where $\widetilde{M}$ is the pullback of the double cover $A \rightarrow A / \mathbf{Z}_{2}$.

Now we need to show that the image of $\tau(m)$ in $T_{*}(A)$ is what we claim it is. To do this, start by making the map $\widetilde{M} \rightarrow D\left(L^{k}\right)$ transverse to the origin; we can do this equivariantly because $\widetilde{M}$ is free [14 or 8]. Let $N$ be the submanifold of points mapping into the origin. As free stable manifolds over $A, \widetilde{M} \rightarrow D\left(L^{k}\right)$ is easily seen to be equivalent to $N \rightarrow *$, or just $N$. Thus the image of $\tau(m)$ in $H_{*}\left(A / \mathbf{Z}_{2}\right)$ is the image of the fundamental class $\left[N / \mathbf{Z}_{2}\right]$.

It suffices to find the image of this fundamental class in $H_{*}(M)$, considering $N / Z_{2}$ to be a submanifold of $M$. But this is actually a familiar problem. Notice that the problem of finding an equivariant map $\widetilde{M} \rightarrow D\left(L^{k}\right)$ transverse to the origin is equivalent to the problem of finding a section $M \rightarrow \widetilde{M} \times{ }_{\mathrm{Z}_{2}} L^{k}$ transverse to the zero-section. In the latter case, $N / Z_{2}$ will be the inverse image of the zero-section. Thus $N / Z_{2}$ is the submanifold of $M$ which is said to be "dual" to the bundle $\xi^{k}$, where $\xi$ is the line bundle associated to the principal bundle $\widetilde{M} \rightarrow M$. It is well known that the image of $\left[N / Z_{2}\right]$ is then the homology class dual to $w_{k}\left(\xi^{k}\right)=w_{1}^{k}(\xi)$, or $w_{1}^{k}(\xi) \cap[M]$. By naturality, the image in $H_{*}\left(A / \mathbf{Z}_{2}\right)$ is $w_{1}^{k} \cap x$ as claimed. 
This result actually gives us all of the transfers that we are interested in. For example,

Corollary 5.2. Suppose that $f: H \Phi \rightarrow \Sigma^{n+1} H \Omega$ is the operation specified by $\sum_{k=0}^{n} a_{k} \theta^{k-n}$, with $a_{k} \in \mathscr{A}_{2}^{k}$ (see the remark after Theorem 2.10), and let $X$ be the fiber of $f$. Then for any free $\mathrm{Z}_{2}-C W$-complex $A$, the transfer

$$
\begin{array}{ccc}
X_{*}\left(A / \mathbf{Z}_{2}\right) & \cong & H_{*}\left(A / \mathbf{Z}_{2}\right) \\
\downarrow \tau & \downarrow \\
X_{*}(A) & \cong & \Sigma^{n} H_{*}\left(A / \mathbf{Z}_{2}\right)
\end{array}
$$

is given by $\tau(x)=\Sigma_{k=0}^{n} w_{1}^{n-k} \cap a_{k} x$, where $w_{1}$ is the first Stiefel-Whitney class of the bundle $A \rightarrow A / \mathbf{Z}_{2}$, and we use the dual action of the Steenrod algebra on $H_{*}\left(A / \mathbf{Z}_{2}\right)$.

Proof. In defining the spectrum $T$, we noted that we had an isomorphism

$$
\left[H \Phi, H\left(\Phi \otimes \mathbf{Z}_{2}\left[\beta_{1}^{-1}\right]\right)\right]_{\mathbf{Z}_{2}}^{*} \cong[H \Phi, \Sigma H \Omega]_{\mathbf{Z}_{2}}^{*} \text {. }
$$

Therefore, $f$ factors through a unique map $H \Phi \rightarrow \Sigma^{n} H\left(\Phi \otimes \mathbf{Z}_{2}\left[\beta_{1}^{-1}\right]\right)$, and we can find a $\mathbf{Z}_{2}$-map $X \rightarrow T$ making the following diagram commute:

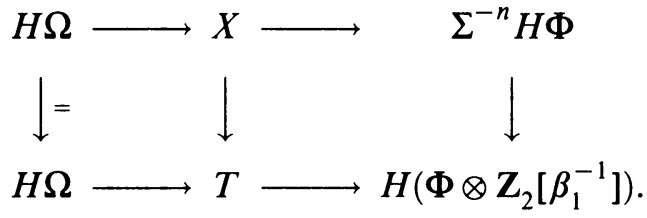

From this we see that we can compute the desired transfer in $X_{*}(-)$ by pushing into $T_{*}(-)$ and computing the transfer there. But $X_{*}\left(A / \mathbf{Z}_{2}\right) \rightarrow T_{*}\left(A / \mathbf{Z}_{2}\right)$ is clearly given by $x \mapsto \Sigma a_{k} x \otimes \beta_{1}^{k-n}$, so the previous result gives us the calculation as claimed.

Most generally,

Corollary 5.3. Suppose that $f: H\left(\Phi \otimes V_{*}\right) \rightarrow \Sigma H\left(\Omega \otimes W_{*}\right)$ is the map corresponding to $f_{*}: V_{*} \rightarrow \mathscr{A}_{2}^{*} \widehat{\otimes} W_{*}\left[\left[\theta^{-1}\right]\right]$, and that $X$ is the fiber of $f$. Then, for any free $\mathrm{Z}_{2}-C W$-complex $A$, the transfer

$$
\begin{gathered}
X_{*}\left(A / \mathbf{Z}_{2}\right) \cong \\
\downarrow \tau \\
X_{*}(A) \cong H_{*}\left(A / \mathbf{Z}_{2}\right) \widehat{\otimes} V_{*} \\
\downarrow
\end{gathered}
$$

is given by $\tau(x \otimes v)=f_{*}(v) x$, where $\mathscr{A}_{2}^{*} \widehat{\otimes} W_{*}\left[\left[\theta^{-1}\right]\right]$ acts on $x$ by the rule

$$
\left(a_{k} \otimes w\right) \theta^{-n} x=\left(w_{1}^{n} \cap a_{k} x\right) \otimes w .
$$

Given the $k$-invariant, we have now written down the transfer. What we would like to do is to reverse this process. That is, we want to say that knowing the transfer determines the $k$-invariant. 
Theorem 5.4. If $f: H \Phi \rightarrow \Sigma^{n+1} H \Omega$ is a nontrivial map, and $X$ is its fiber, then there exists a free $\mathbf{Z}_{2}-C W$-complex $A$ such that the transfer $X_{*}\left(A / \mathbf{Z}_{2}\right) \rightarrow X_{*}(A)$ is nontrivial.

Proof. Suppose that $f$ is specified, in the notation after Theorem 2.10 , by $\Sigma_{k=0}^{n} a_{k} \theta^{k-n}$. By Corollary 5.2, we need to find a free $\mathbf{Z}_{2}$-space $A$ and a class $x \in H_{*}\left(A / \mathbf{Z}_{2}\right)$ such that $\Sigma w_{1}^{n-k} \cap a_{k} x \neq 0$. Equivalently, we need to find a nonequivariant space $B$, a line bundle $\xi$ over it, and a class $x \in H_{*}(B)$ such that $\Sigma w_{1}^{n-k}(\xi) \cap a_{k} x \neq 0$. Dualizing, it suffices to find $B, \xi$, and a class $y \in H^{*}(B)$ such that $\Sigma a_{k}\left(y \cup w_{1}^{n-k}\right) \neq 0$.

Let us take $B$ to be of the form $K \times \mathbf{R P} \mathbf{P}^{\infty}$, where $K$ is any CW-complex, and $\xi$ is taken to be the pullback of the canonical line bundle over $\mathbf{R P}^{\infty}$. $H^{*}\left(K \times \mathbf{R P}^{\infty}\right) \cong H^{*}(K) \otimes \mathbf{Z}_{2}[w]$, where $w=w_{1}(\xi)$. Let $k_{0}$ be the largest index such that $a_{k_{0}}$ is nontrivial. Then, for any $y \in H^{*}(K)$, we have

$$
\begin{aligned}
\sum a_{k}\left(y w^{n-k}\right) & =a_{k_{0}}\left(y w^{n-k_{0}}\right)+\text { higher terms in } w \\
& =a_{k_{0}}(y) w^{n-k_{0}}+\text { higher terms in } w .
\end{aligned}
$$

We can easily choose $K$ and $y$ so that $a_{k_{0}}(y) \neq 0$, and then we are done.

Corollary 5.5. If $f$ and $g$ are two different maps from $H \Phi$ to $\Sigma^{n+1} H \Omega$, and $X$ and $Y$ are their fibers, then there is a free $\mathbf{Z}_{2}$-space $A$ such that the transfers $X_{*}\left(A / \mathbf{Z}_{2}\right) \rightarrow X_{*}(A)$ and $Y_{*}\left(A / \mathbf{Z}_{2}\right) \rightarrow Y_{*}(A)$ are different.

Again, this implies the same result for spectra given by the more general two-stage Postnikov towers of the form $H\left(\Phi \otimes V_{*}\right) \rightarrow \Sigma H\left(\Omega \otimes W_{*}\right)$. In other words, we have shown that these transfers determine the $k$-invariant. This result should be contrasted with Theorem 2.12.

In particular, from Theorem 4.5, we have a better uniqueness result for $m O_{\mathbf{z}_{2}}$ :

Corollary 5.6. $\mathrm{mO}_{\mathbf{Z}_{2}}$ is the only $\mathbf{Z}_{2}$-spectrum which naturally represents geometric bordism and also gives the correct (i.e., the geometrically defined) equivariant transfer for every double cover of the form $A \rightarrow A / Z_{2}$, where $A$ is a free $Z_{2^{-}}$ CW-complex.

Again, to check that $m O_{\mathrm{z}_{2}}^{*}$ does give the correct transfer is an exercise in tracing through the definition of the Pontrjagin-Thom construction. Alternatively, we could apply the formula of Corollary 5.3 above to the known $k$-invariant, and verify that this agrees with the geometry. This is laborious, but instructive in showing another way in which $\Gamma$ arises in studying bordism. 


\section{REFERENCES}

1. J. F. Adams, Prerequisites (on equivariant theory) for Carlsson's lecture, Algebraic Topology, Aarhus 1982, Proceedings, Lecture Notes in Math., vol. 1051, Springer-Verlag, 1984, pp. 483532.

2. J. C. Alexander, The bordism ring of manifolds with involution, Proc. Amer. Math. Soc. 31 (1972), 536-542.

3. J. M. Boardman, Cobordism of involutions revisited, Proc. 2nd Conf. on Comp. Transfer Groups, Part I, Lecture Notes in Math., vol. 298, Springer-Verlag, 1972, pp. 131-151.

4. S. R. Costenoble, Equivariant cobordism and K-theory, Ph. D. Thesis, Univ. of Chicago, 1985.

5. __ The equivariant Conner-Floyd isomorphism, Trans. Amer. Math. Soc. 304 (1987), 801818.

6. __ Unoriented bordism for odd-order groups, Topology Appl. 28 (1988), 277-287.

7. T. tom Dieck, Bordism of G-manifolds and integrality theorems, Topology 9 (1970), 345-358.

8. H. Hauschild, Äquivariante Transversalität und äquivariante Bordismentheorien, Arch. Math. 26 (1975), 536-546.

9. L. G. Lewis, J. P. May and J. E. McClure, Ordinary $R O(G)$-graded cohomology, Bull. Amer. Math. Soc. 4 (1981), 208-212.

10. L. G. Lewis, J. P. May and M. Steinberger, Equivariant stable homotopy theory, Lecture Notes in Math., vol. 1213, Springer-Verlag, 1986.

11. J. P. May and J. E. McClure, A reduction of the Segal conjecture, Current Trends in Algebraic Topology, Canad. Math. Soc. Conf. Proc., vol. 2, Part 2, Amer. Math. Soc., Providence, R. I., 1981, pp. 209-222.

12. R. E. Stong, Notes on cobordism theory, Princeton Univ. Press, 1968.

13. S. Waner, Equivariant $R O(G)$-graded bordism theories, Topology Appl. 17 (1984), 1-26.

14. A. G. Wassermann, Equivariant differential topology, Topology 8 (1969), 127-150.

Department of Mathematics, Hofstra University, Hempstead, New York 11550 